\title{
Genetic and structural basis for SARS-CoV-2 variant neutralization by a two-antibody cocktail
}

\author{
Jinhui Dong ${ }^{1,14}$, Seth J. Zost $\mathbb{1}^{1,14}$, Allison J. Greaney ${ }^{2,3}$, Tyler N. Starr $\mathbb{B}^{2}$, Adam S. Dingens $\mathbb{B}^{2}$, \\ Elaine C. Chen (1) ${ }^{4}$, Rita E. Chen 5,6, James Brett Case ${ }^{10}{ }^{6}$, Rachel E. Sutton', Pavlo Gilchuk1, \\ Jessica Rodriguez', Erica Armstrong1, Christopher Gainza', Rachel S. Nargi', Elad Binshtein ${ }^{1}{ }^{1}$, \\ Xuping Xie ${ }^{7}{ }^{7}$, Xianwen Zhang ${ }^{7}$, Pei-Yong Shi ${ }^{7}{ }^{7}$, James Logue ${ }^{8}{ }^{8}$, Stuart Weston ${ }^{8}$, \\ Marisa E. McGrath ${ }^{8}{ }^{8}$, Matthew B. Frieman ${ }^{8}{ }^{8}$, Tyler Brady ${ }^{9}$, Kevin M. Tuffy ${ }^{9}$, Helen Bright ${ }^{9}$, \\ Yueh-Ming Loo ${ }^{9}$, Patrick M. McTamney ${ }^{9}$, Mark T. Esser ${ }^{9}$, Robert H. Carnahan ${ }^{1,10}$, \\ Michael S. Diamond $\mathbb{B}^{5,6,11,12}$, Jesse D. Bloom $\mathbb{1}^{2,3,13}$ and James E. Crowe Jr $\mathbb{B}^{1,4,10 凶}$
}

\begin{abstract}
Understanding the molecular basis for immune recognition of SARS-CoV-2 spike glycoprotein antigenic sites will inform the development of improved therapeutics. We determined the structures of two human monoclonal antibodies-AZD8895 and AZD1061-which form the basis of the investigational antibody cocktail AZD7442, in complex with the receptor-binding domain (RBD) of SARS-CoV-2 to define the genetic and structural basis of neutralization. AZD8895 forms an 'aromatic cage' at the heavy/light chain interface using germ line-encoded residues in complementarity-determining regions (CDRs) 2 and 3 of the heavy chain and CDRs 1 and 3 of the light chain. These structural features explain why highly similar antibodies (public clonotypes) have been isolated from multiple individuals. AZD1061 has an unusually long LCDR1; the HCDR3 makes interactions with the opposite face of the RBD from that of AZD8895. Using deep mutational scanning and neutralization escape selection experiments, we comprehensively mapped the crucial binding residues of both antibodies and identified positions of concern with regards to virus escape from antibody-mediated neutralization. Both AZD8895 and AZD1061 have strong neutralizing activity against SARS-CoV-2 and variants of concern with antigenic substitutions in the RBD. We conclude that germ line-encoded antibody features enable recognition of the SARS-CoV-2 spike RBD and demonstrate the utility of the cocktail AZD7442 in neutralizing emerging variant viruses.

The current coronavirus disease 2019 (COVID-19) pandemic is caused by SARS-CoV-2, a clade B betacoronavirus (Sarbecovirus subgenus). The spike (S) glycoprotein mediates viral attachment via binding to the host receptor angiotensin-converting enzyme 2 (ACE2) and possibly other host factors, enabling subsequent entry into cells after priming by the host transmembrane protease serine
\end{abstract}

2 (TMPRSS2) ${ }^{1-3}$. The trimeric S glycoprotein consists of two subunits, designated S1 and S2. The S1 subunit binds to ACE2 with its receptor-binding domain (RBD), while the central trimeric S2 subunit functions as a fusion apparatus after the $\mathrm{S}$ glycoprotein sheds the S1 subunit ${ }^{4}$. The human humoral immune response to SARS-CoV-2 has been well documented ${ }^{5-7}$ and many groups have isolated monoclonal antibodies (mAbs) from B cells of previously infected patients who react to the SARS-CoV-2 S protein. A subset of the human mAbs neutralizes virus in vitro and protects against disease in animal models ${ }^{7-17}$. Studies of the human B cell response to SARS-CoV-2 have focused mostly on the S glycoprotein so far due to its critical functions in attachment and entry into host cells ${ }^{7-17}$. For these S glycoprotein-targeting antibodies, the RBD of the S glycoprotein is the dominant target of human neutralizing antibody responses $^{7-17}$. This high frequency of molecular recognition may be related to the accessibility of the RBD to B cell receptors, stemming from a low number of obscuring glycans (only two glycosylation sites on the RBD versus eight or nine sites on the $\mathrm{N}$-terminal domain or S2 subunit, respectively) ${ }^{7}$. The RBD also occupies an apical position and exhibits exposure due to the 'open-closed' dynamics of the $\mathrm{S}$ trimer observed in the $\mathrm{S}$ glycoprotein cryogenic electron microscopy (cryo-EM) structures ${ }^{18-20}$. Potently neutralizing mAbs predominantly target the RBD since this region is directly involved in receptor binding.

In previous studies, we isolated a large panel of human mAbs that bind to the SARS-CoV-2 S glycoprotein from the B cells of patients previously infected with the virus ${ }^{21}$. A subset of these mAbs was shown to bind to recombinant RBD and the S glycoprotein ectodomain and exhibit neutralization activity against SARS-CoV-2 by blocking $S$ glycoprotein-mediated binding to the receptor ${ }^{17}$. Two non-competing antibodies, designated COV2-2196 and COV22130 (later engineered to be long-acting IgG molecules designated

\footnotetext{
'Vanderbilt Vaccine Center, Vanderbilt University Medical Center, Nashville, TN, USA. ²Basic Sciences Division, Fred Hutchinson Cancer Research Center, Seattle, WA, USA. ${ }^{3}$ Department of Genome Sciences \& Medical Scientist Training Program, University of Washington, Seattle, WA, USA. ${ }^{4}$ Department of Pathology, Microbiology, and Immunology, Vanderbilt University Medical Center, Nashville, TN, USA. ${ }^{5}$ Department of Pathology and Immunology, Washington University School of Medicine, St. Louis, MO, USA. ${ }^{6}$ Department of Medicine, Washington University School of Medicine, St. Louis, MO, USA. ${ }^{7}$ Department of Biochemistry \& Molecular Biology, The University of Texas Medical Branch at Galveston, Galveston, TX, USA. ${ }^{8}$ Department of Microbiology and Immunology, The University of Maryland, College Park, MD, USA. ${ }^{9}$ Microbial Sciences, AstraZeneca, Gaithersburg, MD, USA. ${ }^{10}$ Department of Pediatrics, Vanderbilt University Medical Center, Nashville, TN, USA. "Department of Molecular Microbiology, Washington University School of Medicine, St. Louis, MO, USA. ${ }^{12}$ Andrew M. and Jane M. Bursky Center for Human Immunology and Immunotherapy Programs, Washington University School of Medicine, St. Louis, MO, USA. ${ }^{13}$ Howard Hughes Medical Institute, Seattle, WA, USA. ${ }^{14}$ These authors contributed equally: Jinhui Dong, Seth J. Zost. 凶e-mail: james.crowe@vumc.org
} 
as AZD8895 and AZD1061, respectively), synergistically neutralized SARS-CoV-2 in vitro and protected against SARS-CoV-2 infection in mouse models and a rhesus macaque model when used separately or in combination ${ }^{17,21}$. Several phase 3 clinical trials are ongoing to study the antibody cocktail AZD7442, which incorporates AZD8895 and AZD1061, for postexposure prophylaxis (ClinicalTrials.gov ID: NCT04625972), prevention (NCT04625725), outpatient treatment (NCT04723394 and NCT04518410) and inpatient treatment (NCT04501978) of COVID-19. Thus, it is important to define the binding sites of these two antibodies to understand how they interact with the RBD and their ability to neutralize new virus variants.

To understand the atomic details of the recognition of RBD by AZD8895 and AZD1061, we determined the crystal structures of the S glycoprotein RBD in complex with AZD8895 at $2.50 \AA$ (Fig. 1 and Supplementary Table 1) and in complex with both AZD8895 and AZD1061 at $3.00 \AA$ (Fig. 2 and Supplementary Table 1). The substructure of RBD-AZD8895 in the RBD-AZD8895-AZD1061 complex is superimposable with that in the structure of the RBDAZD8895 complex (Extended Data Fig. 1). AZD8895 binds to the receptor-binding ridge of RBD and AZD1061 binds to one side of the RBD edge around residue K444 and the saddle region of the receptor-binding motif (RBM), both partially overlapping the ACE2 binding site (Figs. $1 \mathrm{a}-\mathrm{c}$ and $2 \mathrm{a}, \mathrm{b})$. These features explain the competition between the antibodies and ACE2 for RBD binding from our previous study; for example, both AZD8895 and AZD1061 neutralize the virus by blocking RBD access to the human receptor ACE2 (ref. ${ }^{17}$ ). Aromatic residues from the AZD8895 heavy and light chains form a hydrophobic pocket that surrounds the RBD residue F486 and adjacent residues (G485, N487) (Fig. 1a,d and Extended Data Fig. $2 \mathrm{a}-\mathrm{c}$ ). This mode of antibody-antigen interaction is unusual in that the formation of the antibody pocket is caused by wide spatial separation of the HCDR3 and LCDR3. Overlays of the substructure of RBD in complex with AZD1061 (Fig. 2c) and the structure of RBD in complex with both AZD8895 and AZD1061 (Fig. 2d) suggest that AZD1061 can bind RBD in both 'up' and 'down' conformations of the $S$ trimer. We compared the RBD-AZD1061 crystal structure with the published cryo-EM structures of the human mAbs C119 and C135. AZD1061, C119 and C135 have overlapping but different epitopes since R346 and K444 of the RBD are key residues for AZD1061 and C135 (ref. ${ }^{22}$ ) binding but were not important for C119 binding (Extended Data Fig. 5e).

The AZD8895 interaction with the RBD F486 residue is distinctive. The RBD aromatic residue interacts extensively via a hydrophobic effect and van der Waals interactions with a hydrophobic pocket formed between the AZD8895 heavy and light chains (residue P99 of the heavy chain and an 'aromatic cage' formed by five aromatic side chains) (Fig. 1d and Extended Data Fig. 2a,b). A hydrogen bond (H-bond) network, constructed with 4 direct antibody-RBD H-bonds and 16 water-mediated H-bonds, surround residue F486 and strengthen the antibody-RBD interaction (Extended Data Fig. 2c). Importantly, all residues that interact extensively with the epitope except one (residue P99 of the heavy chain) are encoded by germ line sequences (IGHV1-58*01 and $I G H J 3^{\star} 02$ for the heavy chain and IGKV3-20*01 and IGKJ1 ${ }^{\star} 01$ for the light chain) (Fig. 3a) or only their backbone atoms are involved in the antibody-RBD interactions, such as heavy chain N107 and G99 and light chain S94. We noted another antibody in the literature, S2E12, which is encoded by the same IGHV/IGHJ and IGKV/ $I G K J$ recombinations, with similar but most likely different $I G H D$ genes to those of AZD8895 (IGHD2-15 versus IGHD2-2) (ref. ${ }^{23}$ ). A comparison of the cryo-EM structure of S2E12 in complex with the S glycoprotein (PDB ID: 7K4N) suggests that the mAb S2E12 likely uses nearly identical antibody-RBD interactions as those of AZD8895, although variations in conformations of interface residue side chains can be seen (Extended Data Fig. 2a,b). For example, the phenyl rings of light chain residue Y92 are perpendicular to each other in the two structures. These analyses suggest that AZD8895 and S2E12 have similar modes of recognition of the RBD.

We searched genetic databases to determine if these structural features are present in additional SARS-CoV-2 mAbs isolated by others and found additional members of the clonotype (Fig. 3a). Two other studies reported the same or a similar clonotype of antibodies isolated from multiple patients with COVID-19 who were convalescing ${ }^{13,23}$ and one study found three antibodies with the same IGHV1-58 and IGKV3-20 pairing, without providing information on $\mathrm{D}$ or J gene usage ${ }^{24}$. All these antibodies are reported to bind SARS-CoV-2 RBD avidly and neutralize virus with high pote $\mathrm{ncy}^{13,17,23,24}$. So far, there are only two atomic resolution structures of antibodies encoded by these $\mathrm{V}_{\mathrm{H}^{-}}-\mathrm{D}_{\mathrm{H}^{-}} \mathrm{J}_{\mathrm{H}}$ and $\mathrm{V}_{\mathrm{K}}-\mathrm{J}_{\mathrm{K}}$ recombinations available, the structure for AZD8895 presented in this study and that for S2E12 (ref. ${ }^{23}$ ). We performed homology modelling with the Structuropedia web server ${ }^{25}$ for two additional antibodies of this clonotype from our own panel of anti-SARS-CoV-2 antibodies, designated COV2-2072 and COV2-2381, using the RBD-AZD8895 crystal structure as the template to construct the homology models. As expected, given that these antibodies are members of a shared genetic clonotype, the modelled structures of the RBD-COV2-2072 and RBD-COV2-2381 complexes are virtually superimposable with those of RBD-AZD8895 and RBD-S2E12 at the RBD-antibody interfaces (Extended Data Fig. 3a-e). Additionally, COV2-2072 encodes an $\mathrm{N}$-linked glycosylation sequon in the HCDR3, which we modelled using the GlyProt web server ${ }^{26}$ (Extended Data Fig. 3d). This HCDR3 glycosylation is like that of the recently reported antibody $253 \mathrm{H} 55 \mathrm{~L}^{27}$ and represents an unusual feature for antibodies, given that glycosylation of complementarity-determining regions (CDRs) might adversely affect antigen recognition. However, the AZD8895 structure shows that the disulphide-stapled HCDR3 in this clonotype is angled away from the binding site, explaining how this unusual HCDR3 glycosylation in COV2-2072 can be tolerated without compromising binding (Extended Data Fig. 3d). Inspecting the sequences carefully, we speculate that it is likely that the AZD8895 sequence derives from an independent rearrangement and that COV2-2072 and COV2-2381 derive from a common rearrangement, based on complementary DNA (cDNA) sequences. Differences in the HCDR3 are consistent with this interpretation since differing nucleotide substitutions encoding the same amino acid are present in the two inferred lineages. However, it is impossible to be certain about this for technical reasons. In our original antibody discovery effort, two peripheral blood mononuclear cell samples were pooled to expedite the efficiency of the large-scale screening effort ${ }^{21}$. Thus, these antibodies theoretically could have arisen from two different donors or from two clones that occurred independently in one donor with similar genetic features or from one clone in a single donor that diverged in a complex manner into diverse somatic variants.

We next determined whether we could identify potential precursors of this public clonotype in the antibody variable gene repertoires of circulating B cells from SARS-CoV-2-naïve individuals. We searched for the V-D-J and V-J genes in previously described comprehensive repertoire datasets originating from three healthy human donors without a history of SARS-CoV-2 infection and in datasets from cord blood collected before the COVID-19 pandemic $^{28}$. A total of 386, 193, 47 or 7 heavy chain sequences for this SARS-CoV-2-reactive public clonotype was found in each donor or cord blood repertoire, respectively (Extended Data Fig. 4a). Additionally, we found 516,738 human antibody sequences with the same light chain V-J recombination (IGKV3-20-IGKJ1 ${ }^{\star} 01$ ). A total of 103,534, 191,039 or 222,165 light chain sequences were found for this public clonotype in each donor respectively. Due to the large number of sequences, the top five abundant sequences were aligned from each donor. Multiple sequence alignments were generated for each donor's sequences and logo plots were generated. 
a

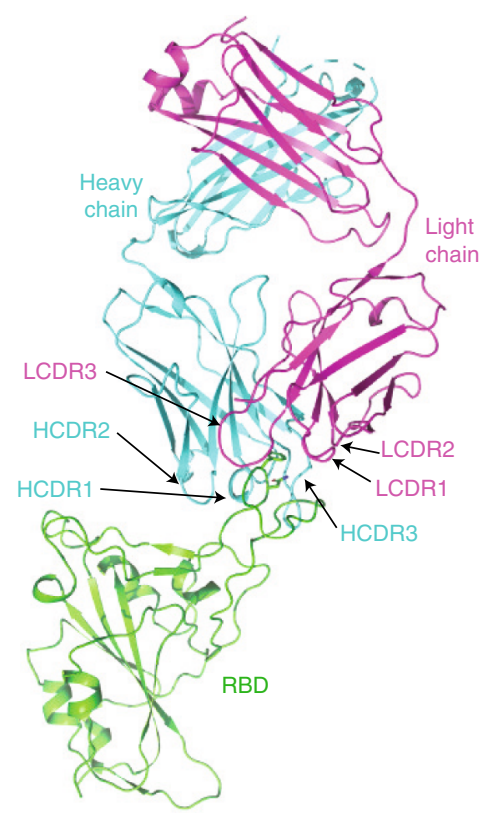

C

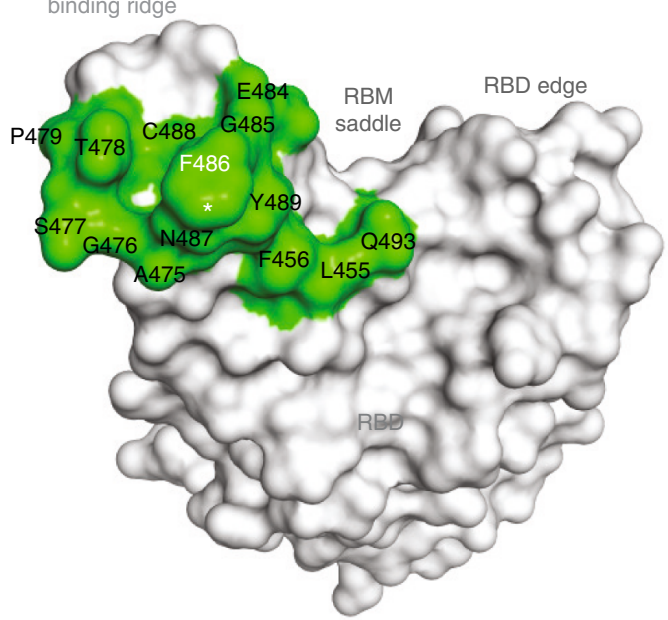

b

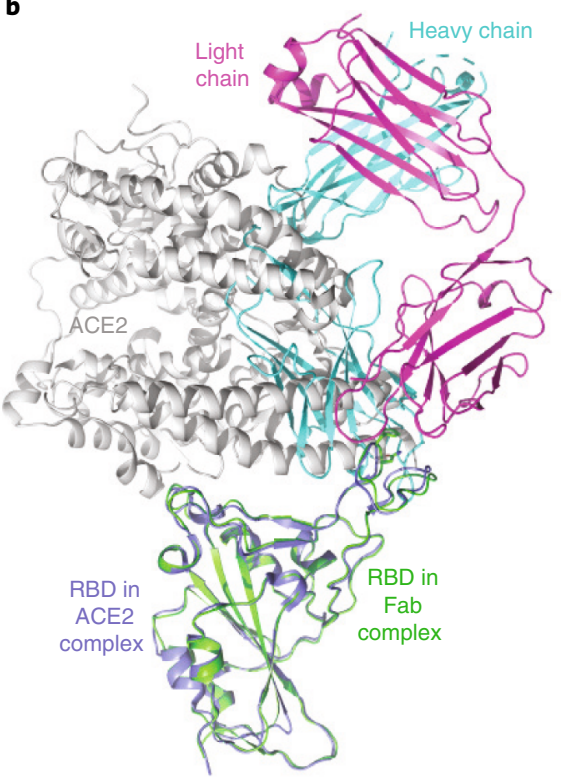

d

AZD8895 CDRs on RBD

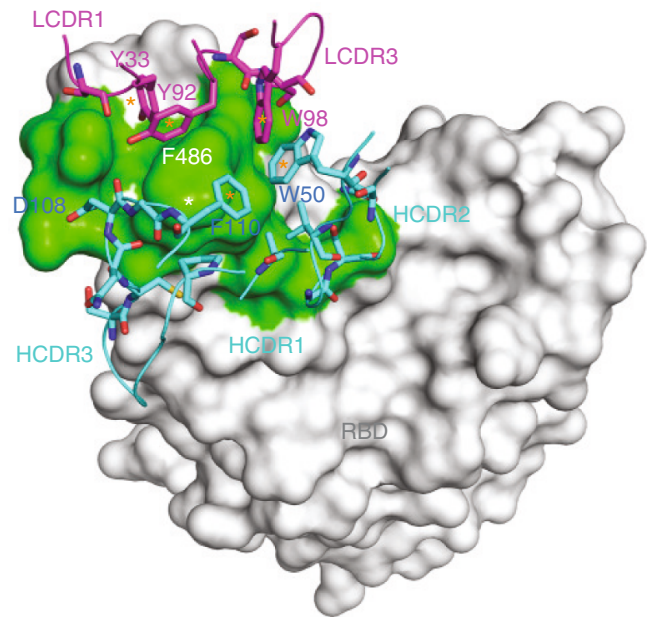

Fig. 1 | Crystal structure of the S glycoprotein RBD in complex with Fab AZD8895. a, Cartoon representation of AZD8895 in complex with RBD. The AZD8895 heavy chain is shown in cyan, the light chain in magenta and the RBD in green. $\mathbf{b}$, The structure of the AZD8895-RBD complex is superimposed onto the structure of the RBD-human ACE2 complex (PDB ID: 6MOJ), using the RBD structure as the reference. The colour scheme of the RBD-AZD8895 complex is the same as that in $\mathbf{a}$. The RBD in the RBD-ACE2 complex is coloured in blue and the human ACE2 peptidase domain in grey. $\mathbf{c}$, Surface representation of the RBD epitope recognized by AZD8895. The epitope residues are coloured in green and labelled in black with the critical contact residue F486 labelled in white. $\mathbf{d}$, Antibody-antigen interactions between AZD8895 and the RBD. The RBD is shown in the same surface representation and orientation as that in $\mathbf{c}$. The AZD8895 paratope residues are shown in stick representation. The heavy chain is coloured in cyan and the light chain is coloured in magenta. The aromatic cage residues Y33, Y92, W98, F110 and W50 are all coloured with darker shades of blue or purple and labelled with an orange star.

The top five sequences with the same recombination event in each donor were identical, resulting in the same logo plots (Extended Data Fig. 4a,b).

We noted that eight of the nine common residues important for RBD binding in the antibody were encoded by germ line gene sequences. Interestingly, these residues were present in all 14 members of the public clonotype that we or others have described (Fig. 3a) $)^{13,17,23,24}$. To validate the importance of these features, we expressed variant antibodies with point mutations in the HCDR3 of the paratope to determine the effect of variation at conserved residues on antibody binding to RBD (Fig. 3b).
We focused site-directed mutagenesis efforts on the P99 and D108 residues since these positions could be impacted at the stage of V-D-J recombination. Altering the D108 residue to A, N or E had little effect but removing the disulphide bond in the HCDR3 through cysteine to alanine substitutions greatly reduced binding. While altering the $\mathrm{P} 99$ residue to $\mathrm{V}$ or $\mathrm{N}$ (observed in other mature antibodies) had little effect, a P99G substitution had a dramatic effect on binding. Additionally, we made two germ line revertants (GRevs) of the AZD8895 antibody. The P99 residue is not templated by the V gene IGHV1-58 nor by the D gene IGHD2-2. However, IGHD2-2 has a likely templated $\mathrm{G}$ at position 99. Therefore, 
a

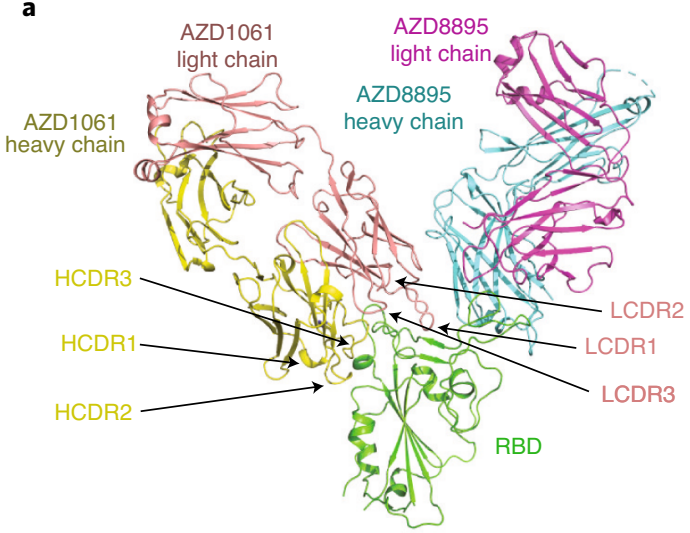

c

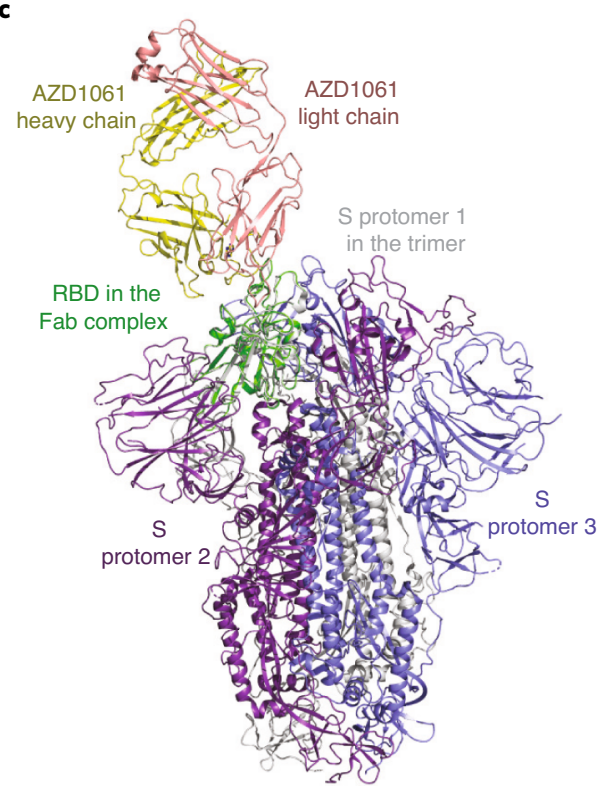

e

AZD1061 binding site on the RBD

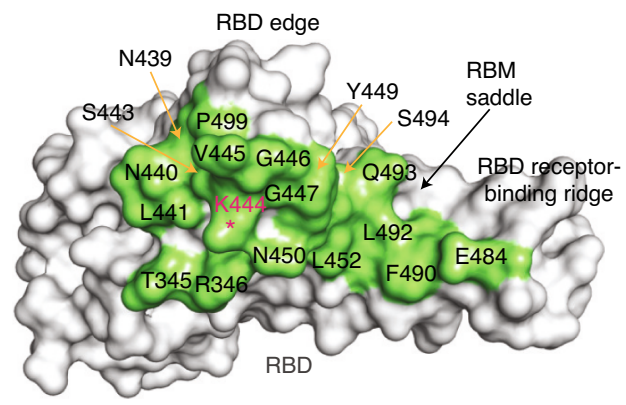

b

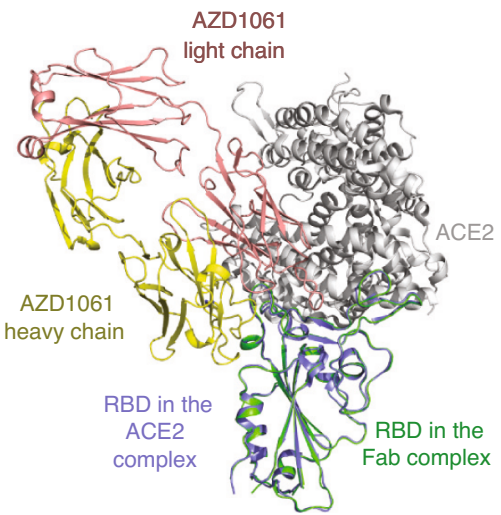

d

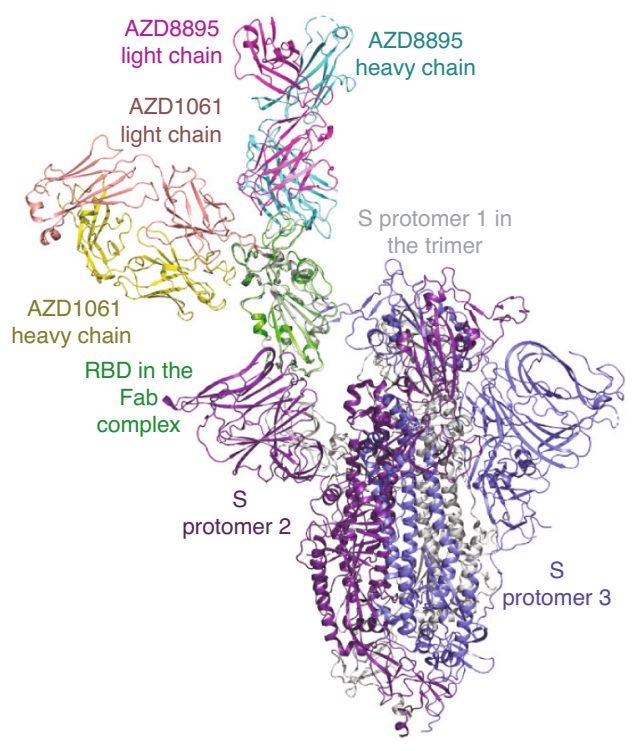

f AZD1061 CDRs on the RBD

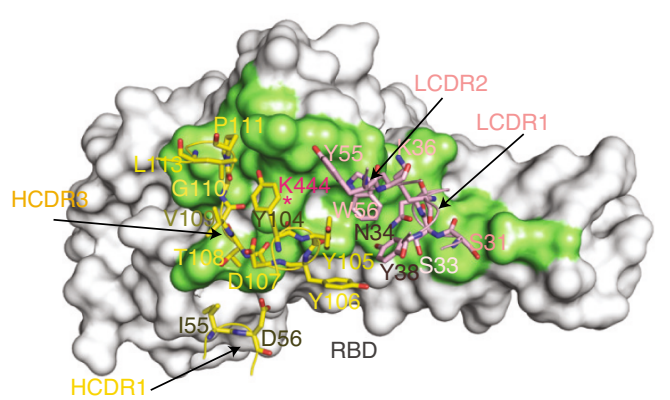

Fig. 2 | Crystal structure of the S glycoprotein RBD in complex with both Fab AZD8895 and Fab AZD1061. a, Cartoon representation of the crystal structure of the S glycoprotein RBD in complex with the AZD8895 and AZD1061 Fabs. The RBD is shown in green, the AZD8895 heavy chain in cyan, the AZD8895 light chain in magenta, the AZD1061 heavy chain in yellow and the AZD1061 light chain in pink. The CDRs of AZD1061 are labelled. $\mathbf{b}$, The structure of the RBD-AZD1061 complex is superimposed onto the structure of the RBD-ACE2 complex (PDB ID: 6MOJ) using the RBD structure as the reference. The colour scheme of the RBD-AZD1061 complex is the same as that in $\mathbf{a}$. The RBD in the RBD-ACE2 complex is coloured in blue and the human ACE2 peptidase domain in grey. $\mathbf{c}$, The structure of the RBD-AZD1061 complex is superimposed onto the structure of S with all RBDs in the 'down' conformation (PDB ID: 6ZOY), using the RBD in one protomer as the reference. The colour scheme of the RBD-AZD1061 complex is the same as that in $\mathbf{a}$. The three protomers of $S$ are coloured in grey, blue or purple, respectively. $\mathbf{d}$, The structure of the RBD-AZD8895-AZD1061 complex is superimposed onto the structure of S with one RBD in the 'up' conformation (PDB ID: 7CAK) using the RBD in the 'up' conformation as the reference. The colour scheme of the RBD-AZD1061 complex is the same as that in $\mathbf{a}$. The three protomers of $S$ are coloured in grey, blue or purple, respectively. $\mathbf{e}$, Surface representation of the RBD epitope recognized by AZD1061. The epitope residues are indicated in green and labelled in black; the key residue K444 is labelled in red. f, Interactions of the AZD1061 paratope residues with the epitope. RBD is shown in the same surface representation and orientation as those in $\mathbf{e}$. The paratope residues are shown in stick representation. The heavy chain is coloured in yellow and the light chain in orange. The key residue K444 is labelled in red. 
a

\begin{tabular}{l} 
Gene $\begin{array}{c}\text { a } \\
\text { IGHV1-58 } \\
\text { /GHD2-2 } \\
\text { /GHD2-8 } \\
\text { IGHD2-15 } \\
\text { IGHJ3*02 }\end{array}$ \\
mAb \\
AZD8895 \\
COV2-2381 \\
COV2-2072 \\
MnC5t2p1_G1 \\
HbnC3t1p2_C6 \\
HbnC3t1p1_C6 \\
\hline S2E12 \\
\hline COV107_P1_53 \\
COV107_P2_81 \\
COV72_P3_42 \\
COV21_P2_F9 \\
COV21_P1_F10 \\
COV57_P1_E6 \\
COV57_P2_H6
\end{tabular}

Heavy chain

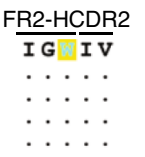

b

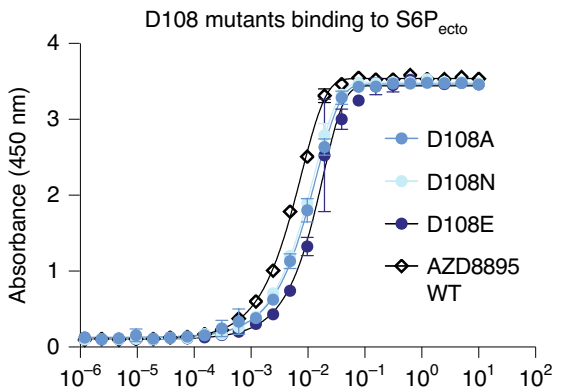

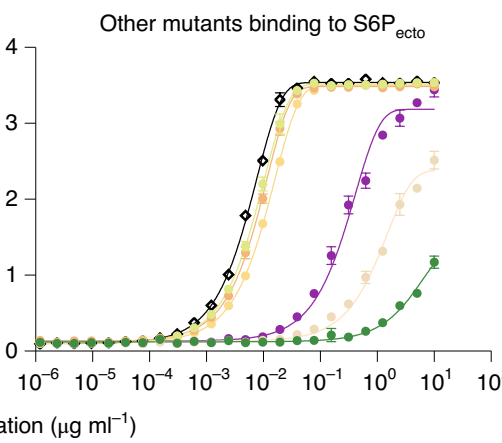

ight chain

\begin{tabular}{|c|c|c|}
\hline LCDR1-FR2 & LCDR3-FR4 & \\
\hline S S Y L A W & 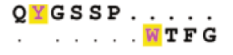 & Reference \\
\hline $\begin{array}{ll}31 & \\
\text { S S } & \text { Y L A W } \\
\text { S S } & \text { I A W } \\
\text { S S } & \text { Y L G W }\end{array}$ & $\left.\begin{array}{l}91 \\
\text { H Y G S S R W TF G } \\
\text { H G S S S Q T T F G } \\
\mathbf{Q} \text { Y G S P. W TFG }\end{array}\right\}$ & Zost et al. ${ }^{17,21}$ \\
\hline $\begin{array}{l}\text { SSYLAW } \\
\text { SSYLAW } \\
\text { S SYLAW }\end{array}$ & $\left.\begin{array}{l}\text { QYGSSP: WTFG } \\
\text { QYGRSP: WTFG } \\
\text { QYGSSP: WFG T T G }\end{array}\right\}$ & Kreer et al. ${ }^{24}$ \\
\hline S SY L A W & QYVGLTGWTFG\} & Tortorici et al. ${ }^{23}$ \\
\hline $\begin{array}{l}\text { S SYLAW } \\
\text { S SYLAW } \\
\text { S SYLAW } \\
\text { S SYLAW } \\
\text { S SYLAW } \\
\text { S SYLAW } \\
\text { S SYLAW }\end{array}$ & 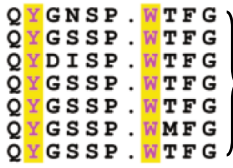 & Robbiani et al. ${ }^{13}$ \\
\hline
\end{tabular}

$\mathrm{mAb}$ concentration $\left(\mu \mathrm{g} \mathrm{ml}^{-1}\right)$

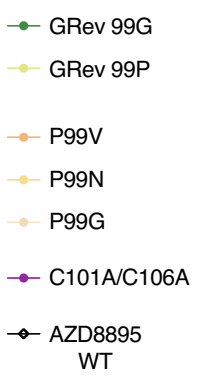

- GRev 99G

Fig. 3 Characterization of important sequence features of the AZD8895 public clonotype. a, IMGT/DomainGapAlign results of the AZD8895 heavy and light chains with germ line V (IGHV1-58 and IGLV3-20), D (IGHD2-2, IGHD 2-8 or IGHD 2-15) or J (IGHJ3*02 and IGKJ1*01) gene segments and with representative variable gene sequences of $m A b s$ in this public clonotype. Key interacting residues and their corresponding residues in germ line genes are highlighted in yellow and coloured in blue except for P99 in purple (heavy chain) or red (light chain). b. Binding curves of point mutants of AZD8895 Mutants of the D108 residue are in blue, GRev of inferred somatic mutations to germ line sequence are in green, P99 mutants are in orange and the C101A/C106A mutations removing the disulphide bond in HCDR3 are in purple. The data points show the mean \pm s.d. for each tested antibody dilution. Experiments were performed in technical triplicate with data shown from a single experiment repeated twice. Data for the AZD8895 WT binding curve shown in both panels are from the same experiment.

two GRevs were tested, one with P99 and the other with G99. Since the P99 residue orients the HCDR3 loop away from the interaction site with antigen, the G99 GRev exhibited reduced binding, whereas the P99 GRev bound antigen equivalently to wild-type (WT) AZD8895 (Fig. 3b).

Unlike AZD8895, AZD1061 uses the HCDR3 for critical contacts. The HCDR3 comprises 22 amino acid residues, which is relatively long for human antibodies. The HCDR3 forms a long, structured loop that is stabilized by short-ranged $\mathrm{H}$-bonds and hydrophobic interactions/aromatic stackings within the HCDR3; it is further strengthened by its interactions ( $\mathrm{H}$-bonds and aromatic stackings) with residues of the light chain (Extended Data Fig. 5a,b). The AZD1061 heavy or light chain is encoded by the germ line gene IGHV3-15 or IGKV4-1, respectively; the two genes encode the longest germ line-encoded HCDR2 (10 residues) and LCDR1 (12 residues) loops. The heavy chain V-D-J recombination, HCDR3 mutations and the pairing of heavy and light chains result in a binding cleft between the heavy and light chains, matching the shape of the RBD region centred at the S443-Y449 loop (Fig. $2 \mathrm{a}$ and Extended Data Fig. 5c). Closely related to these structural features, only HCDR3, LCDR1, HCDR2 and LCDR2 are involved in the formation of the paratope (Fig. 2e,f and Extended Data Fig. $2 \mathrm{e}, \mathrm{f})$. Inspection of the antibody-RBD interface reveals a region that likely drives much of the energy of the interaction. The RBD residue K444 side chain is surrounded by subloop Y104-V109 of the HCDR3 loop and the positive charge on the side chain nitrogen atom is neutralized by the HCDR3 residue D107 side chain, three main chain carbonyl oxygen atoms from Y105, D107 and V109 and the electron-rich face of the Y104 phenyl ring (cation- $\pi$ interaction) (Extended Data Fig. 2e). In addition, the AZD1061 light chain LCDR1 and LCDR2 make extensive contacts with the RBD (Extended Data Fig. 2f). In the crystal structure of the RBD in complex with both AZD8895 and AZD1061, we noted that the closely spaced AZD8895 and AZD1061 fragment antigen-binding (Fab) may interact directly with each other when bound to RBD (Extended Data Fig. 6).

To better understand the RBD residues critical for the binding of AZD8895 and AZD1061, we used a deep mutational scanning (DMS) approach to map all RBD mutations that escape antibody binding ${ }^{29}$ (Extended Data Fig. 7). Antibody escape in the DMS studies was quantified as an 'escape fraction' ranging from 0 (no cells with the mutation in the antibody escape bin) to 1 (all cells with the mutation in the antibody escape bin). For both antibodies, we identified several key positions, nearly all in the antibody binding site, where RBD mutations strongly disrupted binding (Fig. 4a-d). We leveraged our previous work quantifying the effects of RBD mutations on ACE2 binding ${ }^{30}$ to overlay the effect on ACE2 binding for mutations that abrogated antibody binding to the Wuhan-1 


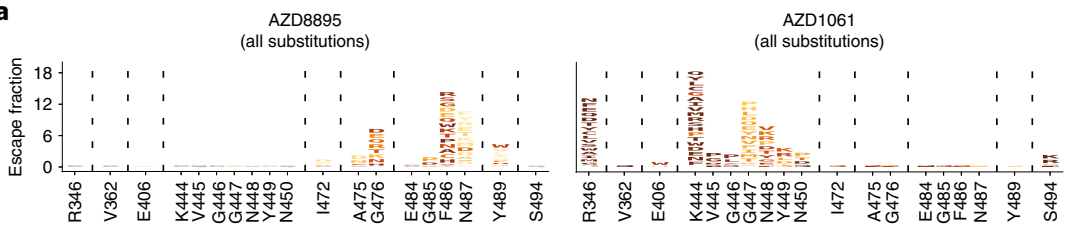

b
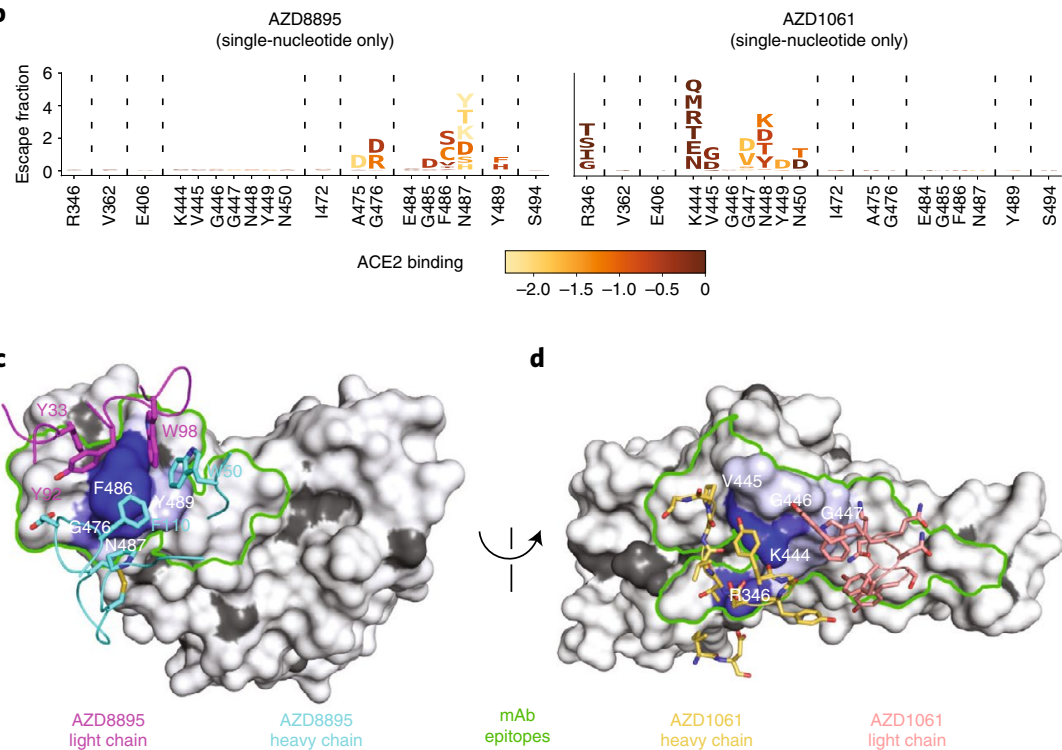

d

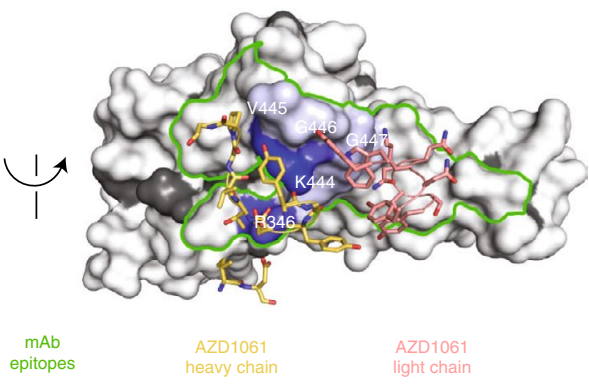

\begin{tabular}{|c|c|c|}
\hline $\begin{array}{l}\text { Selection } \\
\text { condition }\end{array}$ & $\begin{array}{l}\text { Replicates with } \\
\text { escape/total }\end{array}$ & $\begin{array}{l}\text { Mutations } \\
\text { identified }\end{array}$ \\
\hline AZD8895 & $0 / 88(0 \%)$ & Not applicable \\
\hline AZD1061 & $8 / 20(40 \%)$ & $\mathrm{K} 444 \mathrm{R}(6), \mathrm{K} 444 \mathrm{E}(2)$ \\
\hline AZD7442 & 0/104 (0\%) & Not applicable \\
\hline
\end{tabular}

f SARS-CoV-2 resistant variant selection

\begin{tabular}{|c|c|c|c|}
\multicolumn{1}{c}{$\begin{array}{c}\text { Passaging } \\
\text { condition }\end{array}$} & \multicolumn{1}{c}{$\begin{array}{c}\text { Test } \\
\text { condition }\end{array}$} & \multicolumn{1}{c}{$\begin{array}{c}\text { Resistance } \\
\text { detected }\end{array}$} & $\begin{array}{c}\text { Mutations } \\
\text { identified }\end{array}$ \\
\hline AZD8895 & AZD8895 & No & Not applicable \\
& AZD7442 & No & N74K, R346I, S686G \\
\hline AZD1061 & AZD1061 & Yes & Not applicable \\
\hline AZD7442 & AZD7442 & No & Not applicable \\
\hline
\end{tabular}

h

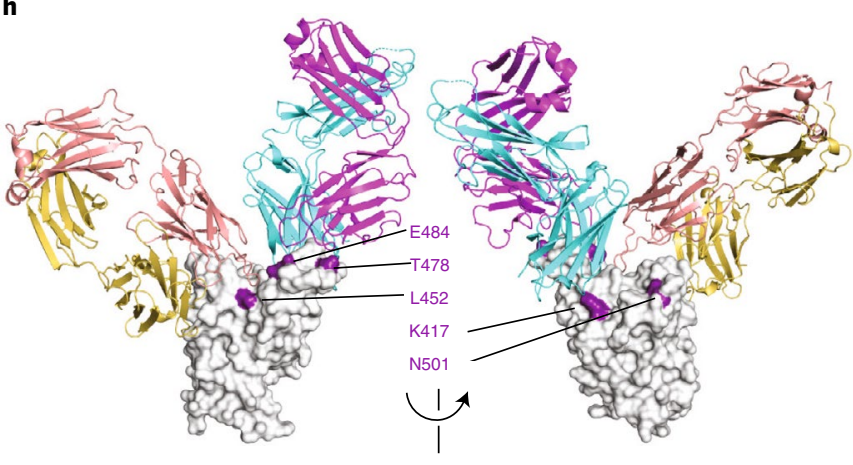

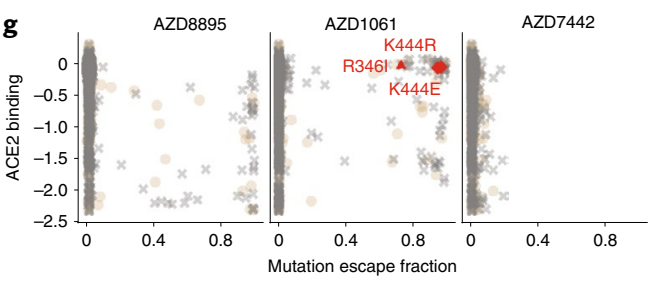

Single-nucleotide $\quad \times$ Multi-nucleotide

- VSV-SARS-CoV-2 selected A SARS-CoV-2 selected

i

i SARS-CoV-2 viruses

\begin{tabular}{|c|c|c|c|c|c|c|c|c|}
\hline \multirow[b]{2}{*}{ RBD mutations } & D614G & $\begin{array}{c}\mathrm{N} 501 Y_{+} \\
\text {D614G }\end{array}$ & $\begin{array}{l}\text { B.1.1.7 } \\
\text { (alpha) }\end{array}$ & $\begin{array}{c}\text { Wash- } \\
\text { B.1.351 } \\
\text { (beta) }\end{array}$ & $\begin{array}{c}\text { Wash- } \\
\text { B.1.1.28 } \\
\text { (gamma) }\end{array}$ & $\begin{array}{l}\text { B.1.429 } \\
\text { (epsilon) }\end{array}$ & $\begin{array}{c}\text { B.1.617.1 } \\
\text { (kappa) }\end{array}$ & $\begin{array}{l}\text { B.1.617.2 } \\
\text { (delta) }\end{array}$ \\
\hline & - & N501Y & N501Y & $\begin{array}{l}\text { K417N } \\
\text { E484K } \\
\text { N501Y }\end{array}$ & $\begin{array}{l}\text { K417T } \\
\text { E484K } \\
\text { N501Y }\end{array}$ & L452R & $\begin{array}{l}\text { L452R } \\
\text { E484Q }\end{array}$ & $\begin{array}{l}\text { L452R } \\
\text { T478K }\end{array}$ \\
\hline $\mathrm{mAb}$ & \multicolumn{8}{|c|}{$\begin{array}{l}\text { mAb neutralizing activity }\left(\mathrm{IC}_{50} ; \mathrm{ng} \mathrm{m}^{-1}\right) \\
\text { for the indicated virus }\end{array}$} \\
\hline AZD8895 & 4 & 6 & 6 & 25 & 46 & 5 & 2 & 2 \\
\hline AZD1061 & 28 & 37 & 10 & 22 & 10 & 75 & 14 & 43 \\
\hline AZD7442 & 4 & 7 & 4 & 15 & 8 & 14 & 2 & 3 \\
\hline
\end{tabular}

Fig. 4 | Critical residues for AZD8895 and AZD1061 binding. a, Logo plots of mutation escape fractions at RBD sites with strong escape for AZD8895 (left) or AZD1061 (right). Taller letters indicate greater escape. Mutations are coloured by the degree to which they reduced RBD binding to human ACE2. Data shown are the average of two independent escape experiments using two independent yeast libraries; correlations are shown in Extended Data Fig. 7b,c. Interactive plots can be found at https://jbloomlab.github.io/SARS-CoV-2-RBD_MAP_AZ_Abs/.b, Logo plots of mutation escape fractions that are accessible by single-nucleotide substitutions from the Wuhan-1 reference strain. Effect represented as in a. c, Mapping DMS escape mutations for AZD8895 onto the RBD surface. Blue, RBD site with the greatest cumulative antibody escape; white, no escape detected; grey, residues where deleterious effects on RBD expression prevented assessment. Heavy chain, cyan; light chain, magenta. Two replicates were performed with independent libraries, as described in a. Green, structurally defined AZD8895 footprint on RBD. d, Mapping DMS escape mutations for AZD1061 onto the RBD surface in the RBD-AZD1061 complex. Mutations that abrogated AZD1061 binding are displayed on the RBD structure using a heatmap as in c. Heavy chain, yellow; light chain, pink; green, structurally defined AZD1061 footprint on RBD. e, Table showing the results of the VSV-SARS-CoV-2 escape selection experiments. f, Table showing the results of the passage of SARS-CoV-2 in the presence of subneutralizing concentrations of mAbs. $\mathbf{g}$, Scatter plot showing the DMS data from $\mathbf{a}$, with the mutation escape fraction on the $x$ axis and the effect on ACE2 binding on the $y$ axis. Crosses, mutations accessible only by multi-nucleotide substitutions; circles, mutations accessible by single-nucleotide substitutions. The substitutions selected by AZD1061 in VSV-SARS-CoV-2 (K444R, K444E) or authentic SARS-CoV-2 (R346I) are denoted. h, Locations of mutations in VOCs/VOIs mapped onto the RBD/AZD8895/AZD1061 crystal structure. i, Neutralization (FRNT) against SARS-CoV-2 VOC/VOIs. Assays were performed in duplicate and repeated twice. Data are the mean of two independent experiments. Wash-B.1.351 refers to a chimeric recombinant virus with the WA1/2020 backbone expressing the B.1.351 (beta) S-gene; Wash-B.1.1.28 refers to a similar virus expressing the B.1.1.28/P1 (gamma) S-gene.

strain RBD (Fig. 4a,b). For AZD8895, many mutations to F486 and N487 had escape fractions approaching 1 (that is, all cells expressing this RBD mutation fell into the antibody escape bin), reinforcing the importance of the contributions of these two residues to antibody binding. Similarly, for AZD1061, mutation of residue K444 to any of the other 19 amino acids abrogated antibody binding, 
indicating that the lysine at this position is critical for the antibodyRBD interaction.

Nevertheless, few antibody binding site residues were identified as sites where mutations greatly reduced binding. Several explanations are possible: (1) some binding site residues may not be critical for binding; (2) some RBD residues do not use their side chains to form interactions with the mAbs; or (3) mutations at some sites may not be tolerated for RBD expression ${ }^{30}$. For instance, residues L455, F456 and Q493 are part of the structurally defined binding site for AZD8895 (Fig. 1d) but mutations to these sites did not impact antibody binding (Fig. 4a), suggesting that these residues do not make critical binding contributions. Superimposition of the RBDAZD8895 complex onto the RBD-S2E12 complex clearly demonstrates a flexible hinge region between the $\mathrm{RBD}$ ridge and the rest of the RBD that is maintained when antibody is bound (Extended Data Fig. 2d). This finding indicates that mutations at these three positions could be well tolerated for antibody-RBD binding and supports the non-essential nature of these residues for AZD8895 or S2E12 binding.

Importantly, AZD8895 and AZD1061 do not compete with one another for binding to the $\mathrm{RBD}^{17}$, suggesting they could comprise an escape-resistant cocktail for prophylactic or therapeutic use. Indeed, the binding sites and escape variant maps for these two antibodies are non-overlapping. To test whether there were single mutations that could escape binding of both antibodies, we performed escape variant mapping experiments with AZD7442 but we did not detect any mutation that had an escape fraction $>0.2$, indicating only partial reduction in antibody binding, whereas the individual amino acid mutations with the largest effects for each of the single antibodies was approximately 1 , indicating substantial loss of antibody binding due to that amino acid change (Extended Data Fig. 7d).

Although these experiments map all individual amino acid mutations that escape antibody binding to the RBD, we also sought to determine which mutations have the potential to arise during viral growth. To address this question, we first attempted to select escape mutations using a recombinant vesicular stomatitis virus (VSV) expressing the SARS-CoV-2 S glycoprotein (VSV-SARS-CoV-2); ${ }^{31}$ (Fig. 4e). We expected that the only amino acid mutations that would be selected during viral growth were those (1) arising by single-nucleotide RNA changes, (2) causing minimal deleterious effect on ACE2 binding and expression and (3) substantially impacting antibody binding ${ }^{29,30}$. Indeed, we did not detect any AZD8895-induced mutations that were both single-nucleotide accessible and relatively well tolerated with respect to effects on ACE2 binding (Fig. 4b), which may explain why escape mutants were not selected in any of the 88 independent replicates of recombinant VSV growth in the presence of antibody (Fig. 4e and Extended Data Fig. 7g). For AZD1061, mutations to site K444, a site that is relatively tolerant to mutation ${ }^{30}$, demonstrated the most frequent escape from antibody binding in neutralization assays with the VSV chimeric virus. K444R (selected in 6 out of 20 replicates) or K444E (selected in 2 out of 20 replicates) were identified in $40 \%$ of the replicates of recombinant VSV growth in the presence of AZD1061 (Fig. 4e and Extended Data Fig. 7g).

To explore resistance with authentic infectious virus, SARS-CoV-2 strain USA-WA1/2020 was passaged serially in Vero cell cultures with AZD8895, AZD1061 or AZD7442 at concentrations beginning at their respective half-maximal inhibitory concentration $\left(\mathrm{IC}_{50}\right)$ values and increased stepwise to their $\mathrm{IC}_{90}$ value with each passage (Extended Data Fig. 8a). As a control, virus was passaged in the absence of antibody. After the final passage, viruses were evaluated for susceptibility against the partner antibody at a final concentration of $10 \times$ the $\mathrm{IC}_{90}$ concentration by plaque assay. We did not detect any plaques resistant to neutralization by AZD8895 or the AZD7442 cocktail. Virus that was passaged serially in the presence of AZD1061 formed plaques to a titre of $1.2 \times 10^{7}$ plaque-forming units (PFUs) $\mathrm{ml}^{-1}$ after selection in $10 \times$ the $\mathrm{IC}_{90}$ value concentration of AZD1061, but plaques were not formed in the presence of AZD7442. Plaques $(n=6)$ were selected randomly and the phenotype of the escaped viruses was characterized in virus neutralization tests (Extended Data Fig. 8b). The S-gene form plaque isolate was amplified and sequenced, revealing the same three amino acid changes in all six plaques: N74K, R346I and S686G (Fig. 4f). The S686G change in SARS-CoV-2 has been reported previously to be associated with serial passaging in Vero cells ${ }^{32}$ and was identified in SARS-CoV-2 isolated from infected ferrets ${ }^{33}$ or non-human primates $^{34}$ and it is predicted to decrease furin activity ${ }^{32}$. The N74K residue is located in the N-terminal domain outside the AZD1061 binding site and results in the loss of a glycan ${ }^{35}$. The R346I residue is located in the binding site of AZD1061 and may be associated with AZD1061 resistance. In a biolayer interferometry-based binding test, AZD1061 retained binding to S glycoproteins with N74K and S686G to levels like the reference $S$ glycoprotein (Extended Data Fig. $8 \mathrm{c}, \mathrm{d})$. However, there were dramatic reductions in AZD1061 binding to S glycoproteins containing either the R346I substitution or the K444R or K444E substitutions selected in the VSV-SARS-CoV-2 virus (Extended Data Fig. 8c,d). Importantly, AZD8895 binding was unaffected by these substitutions (Extended Data Fig. 8c,d). The data confirmed that N74K and S686G were mutations that accumulated during the passaging of SARS-CoV-2 in Vero cells and that $\mathrm{R} 346 \mathrm{I}$ is the primary amino acid change in RBD that contributed to escape from AZD1061. The K444R and K444E substitutions selected in the VSV-SARS-CoV-2 system and the R346I substitution selected by passage with authentic SARS-CoV-2 are accessible by single-nucleotide substitution and preserve ACE2 binding activity (Fig. 4g), indicating that our DMS analysis using yeast predicted the mutations in SARS-CoV-2 selected in the presence of the AZD1061 antibody. Taken together, these results comprehensively map the effects of all amino acid substitutions on the binding of AZD8895 and AZD1061 and identify sites of possible concern for viral evolution. That said, variants containing mutations at residues K444 and R346 are rare among all sequenced viruses present in the Global Initiative on Sharing Avian Influenza Data databases (all $\leq 0.08 \%$ when accessed on 11 May 2021).

Viral variants of concern (VOCs) with increased transmissibility and antigenic mutations have been reported in clinical isolates. Due to the potential of these VOCs to undermine the protective effects of immunity induced by infection, vaccination or $\mathrm{mAb}$ therapeutics, VOCs are the subject of intense study ${ }^{36-41}$. We next sought to determine whether some of the amino acid substitutions present in these emerging VOCs affect the activity of these potently neutralizing antibodies in focus reduction neutralization tests $^{42}$. We tested authentic SARS-CoV-2 viruses that contained S glycoproteins matching the B.1.1.7 (alpha), B1.1.351 (beta), B.1.1.28 (gamma), B.1.617.1 (kappa) or B.1.617.2 (delta) VOCs or the B.1.429 (epsilon) virus of interest (VOI). Substitutions at position E484 were of special interest since this residue is located within $5 \AA$ of each of the mAbs in the complex of Fabs and RBD, albeit at the very peripheral edge of the binding sites. E484K is present in the emerging lineages B.1.351 (ref. ${ }^{38}$ ) and B.1.1.28 (ref. ${ }^{39}$ ), along with substitutions at $\mathrm{K} 417$, and has been demonstrated to alter the binding of some $\mathrm{mAbs}^{40,43,44}$ as well as human polyclonal serum antibodies $^{45}$. Variants containing E484K also have been shown to be neutralized less efficiently by convalescent serum and plasma from SARS-CoV-2 survivors ${ }^{46-48}$. An E484Q substitution is present in the B.1.617.1 (kappa) VOC, which has also been shown to have reduced susceptibility to neutralization ${ }^{40}$. Additionally, an L452R substitution is present in the B.1.617.1 and B.1.617.2 (ref. ${ }^{40}$ ) VOCs and the B.1.429 (ref. ${ }^{41}$ ) VOI, which also abrogates the binding of some neutralizing antibodies. B.1.617.2 contains an additional T478K mutation in the $\mathrm{RBD}^{40}$. AZD8895, AZD1061 and AZD7442 
all potently neutralized representatives of the B.1.1.7, B.1.351, B.1.1.28, B.1.617.1 and B.1.617.2 VOCs and B.1.429 VOI, with $\mathrm{IC}_{50}$ values comparable to those for the D614G strain, with at most a 6- to 11-fold reduction for AZD8895 in 2 cases (Fig. 4h,i). Recent reports from others described neutralization data for recombinant IgG incorporating the Fv of COV2-2196 and COV2-2130, the predecessors of the engineered long-acting AZD8895 and AZD1061 antibodies, based on the Fv sequences we published previously. Those studies also showed that amino acid substitutions present in B.1.1.7, B.1.351 or P.1 (B.1.1.28) VOCs had little effect on the neutralization of these $\mathrm{mAbs}^{42,49-52}$.

\section{Discussion}

The process of B cell development, where diverse variable gene segments are recombined, results in human naïve $B$ cell repertoires containing an enormous amount of structural diversity in the CDRs of the antibodies they encode. Despite this extensive and diverse pool of naïve B cells, infection or vaccination with viral pathogens sometimes elicits antibodies in diverse individuals that share common structural features encoded by the same antibody variable genes. Examples of recurring variable gene usage have been described for antibody responses to human rotavirus ${ }^{16,53}$, human immunodeficiency virus ${ }^{54-57}$, influenza $A$ virus ${ }^{58-61}$ and hepatitis $\mathrm{C}$ virus $^{62,63}$, among others. The recognition of the use of common variable genes in antiviral responses has led to the general concept of B cell public clonotypes or B cells with similar genetic features in their variable regions that encode for antibodies with similar patterns of specificity and function in different individuals. Several recent reports described the identification of public clonotypes in the antibody responses to SARS-CoV-2 (refs. ${ }^{8,13,64,65}$ ). Identifying and understanding the genetic and structural basis for selection of public clonotypes is valuable since this information forms the central conceptual underpinning for many current rational, structure-based vaccine design efforts ${ }^{66}$. Our structural analyses define the molecular basis for the frequent selection of a public clonotype of human antibodies sharing heavy chain V-D-J and light chain V-J recombinations that target the same region of the SARS-CoV-2 S RBD. Germ line antibody gene-encoded residues in heavy and light chains play a vital role in antigen recognition, suggesting that few somatic mutations are required for antibody maturation of this clonotype. The existence of potently neutralizing public clonotypes across multiple individuals may in part account for the remarkable efficacy of S glycoprotein-based vaccines that is being observed in the clinic. One might envision an opportunity to elicit serum neutralizing antibody titres with even higher neutralization potency using domain- or motif-based vaccine designs for this antigenic site to prime human immune responses to elicit this clonotype.

The recent emergence of variant virus lineages with increased transmissibility and altered sequences in known sites of neutralization is concerning for the capacity of SARS-CoV-2 to evade current antibody countermeasures in development and testing. Our comprehensive mapping of the effect of RBD mutations on the binding of AZD8895 and AZD1061 underscores their use as a rationally designed cocktail, given that they have different escape mutations. The data from our DMS experiments are also consistent with the binding sites determined by our antibody-RBD crystal structures and the DMS results predict the mutations present in resistant variants selected by in vitro passaging experiments. We tested the activity of the individual antibodies or the cocktail against authentic viruses containing mutations from several important SARS-CoV-2 VOCs and demonstrated that the individual antibodies or their combination are capable of potently neutralizing these emerging variants. Recent work from others has also demonstrated that some circulating VOCs exhibit substantial escape from neutralization of many human mAbs in clinical development; however, AZD8895 and AZD1061 still potently neutralized viruses that included the B.1.1.7, B.1.351, B.1.1.28, B.1.617.1 and B.1.617.2 VOCs and B.1.429 VOI. While other VOIs have been described containing $\mathrm{N} 439 \mathrm{~K}^{67}$, S477N ${ }^{44}$ or $\mathrm{F} 486$ (ref. ${ }^{68}$ ), substitutions with potential antigenic effects, our DMS experiments suggest that these mutations are not likely to have substantial effects on the binding and neutralization of AZD8895, AD1061 or AZD7442. Taken together, this work defines the molecular basis for potent neutralization of SARS-CoV-2 by AZD8895 and AZD1061 and demonstrates that these antibodies efficiently neutralize emerging antigenic variants either separately or in combination, underscoring the promise of the AZD7442 investigational cocktail for use in the prevention and treatment of COVID-19.

\section{Methods}

Expression and purification of recombinant RBD of the SARS-CoV-2 S glycoprotein. The DNA segments corresponding to the S glycoprotein RBD (residues 319-528) was sequence-optimized for expression, synthesized and cloned into the pTwist-CMV expression DNA plasmid downstream of the interleukin-2 signal peptide (MYRMQLLSCIALSLALVTNS) (Twist Bioscience). A three-amino acid linker (GSG) and a histidine tag were incorporated at the $\mathrm{C}$ terminus of the expression constructs to facilitate protein purification. Expi293F cells were transfected transiently with the plasmid encoding RBD and culture supernatants were collected after $5 \mathrm{~d}$. RBD was purified from the supernatants by nickel affinity chromatography with HisTrap Excel columns (GE Healthcare Life Sciences). For the protein production used in the crystallization trials, $5 \mu \mathrm{M}$ of kifunensine was included in the culture medium to produce RBD with high mannose glycans. The high mannose glycoproteins were subsequently treated with endoglycosidase F1 (Merck Millipore) to obtain homogeneously deglycosylated RBD.

Expression and purification of recombinant AZD8895 and AZD1061 Fabs. The DNA fragments corresponding to the AZD8895 and AZD1061 heavy chain variable domains with human IgG1 CH1 domain and light chain variable domains with human kappa chain constant domain were synthesized and cloned into the pTwist vector (Twist Bioscience). This vector includes the heavy chain of each Fab, followed by a GGGGS linker, a furin cleavage site, a T2A ribosomal cleavage site and the light chain of each Fab. Expression of the heavy and light chains is driven by the same cytomegalovirus promoter. AZD8895 and AZD1061 Fabs were expressed in ExpiCHO cells by transient transfection with the expression plasmid. The recombinant Fab was purified from the culture supernatant using an anti-CH1 CaptureSelect column (Thermo Fisher Scientific). For the RBDAZD8895 complex, the WT sequence of AZD8895 was used for expression. For the RBD-AZD8895-AZD1061 complex, a modified version of AZD8895 Fab was used where the first two amino acids of the variable region were mutated from QM to EV.

Crystallization and structural determination of antibody-antigen complexes. Purified AZD8895 Fab was mixed with deglycosylated RBD in a molar ratio of $1: 1.5$ and the mixture was purified further by size-exclusion chromatography with a Superdex-200 Increase column (GE Healthcare Life Sciences) to obtain the antibody-antigen complex. To obtain the RBD-AZD8895-AZD1061 triple complex, purified and deglycosylated RBD was mixed with both AZD8895 and AZD1061 Fabs in a molar ratio of 1:1.5:1.5 and the triple complex was purified with a Superdex-200 Increase column. The complexes were concentrated to about $10 \mathrm{mg} \mathrm{ml}^{-1}$ and subjected to crystallization trials. The RBD-AZD8895 complex was crystallized in $16-18 \%$ polyethylene glycol 3350, 0.2 Tris- $\mathrm{HCl}, \mathrm{pH}$ 8.0-8.5 and the RBD-AZD8895-AZD1061 complex was crystallized in 5\% (w/v) polyethylene glycol 1000, $100 \mathrm{mM}$ of sodium phosphate dibasic/citric acid, $\mathrm{pH}$ $4.2,40 \%(\mathrm{v} / \mathrm{v})$ reagent alcohol. Cryoprotection solution was made by mixing crystallization solution with $100 \%$ glycerol in a volume ratio of 20:7 for crystals of both complexes. Protein crystals were flash-frozen in liquid nitrogen after a quick soaking in the cryoprotection solution. Diffraction data were collected at $100 \mathrm{~K}$ at the beamline 21-ID-F (wavelength: $0.97872 \AA$ ) for the RBD-AZD8895 complex and 21-ID-G (wavelength: $0.97857 \AA$ ) for the RBD-AZD8895-AZD1061 complex at the Advanced Photon Source. Diffraction data were processed with XDS version 31 January $2020^{69}$ and CCP4 suite version $7.1^{70}$. The crystal structures were solved by molecular replacement using the structure of RBD in complex with Fab CC12.1 (PDB ID: 6XC2) and Fab structure of MR78 (PDB ID: 5JRP) with the program Phaser version $2.8 .3^{71}$. The structures were refined and rebuilt manually with Phenix version $1.14^{72}$ or Coot version $0.9 .3^{73}$, respectively. The Ramachandran statistics for the final structure of RBD-AZD8895 were: 95.82\% favoured; $4.18 \%$ allowed; and $0.00 \%$ disallowed. The Ramachandran statistics for the final structure of RBD-AZD8895-AZD1061 were: $95.34 \%$ favoured; $4.37 \%$ allowed; and $0.00 \%$ disallowed. The models have been deposited with the Protein Data Bank. PyMOL v.1.8 (Schrödinger) was used to make all the structural figures. 
AZD8895 mutant generation. Structurally important residues in the AZD8895 heavy chain sequence were identified as D108, P99 and the disulphide bond in HCDR3. The D108 residue was mutated to alanine, asparagine and glutamic acid. The $\mathrm{P} 99$ residue was mutated to valine, asparagine and glycine. The disulphide bond was removed by introducing C101A/C106A mutations. Additionally, the GRev forms of AZD8895 were generated by aligning the sequence to the identified germ line sequences using IGBLAST and reverting the residues that were not germ line-encoded. DNA fragments corresponding to the AZD8895 mutant heavy chain variable domains with human IgG1 and light chain variable domain with human kappa chain constant domain were synthesized and cloned into the pTwist_mCis vector (Twist Bioscience) as described previously ${ }^{21}$. Constructs were transformed into Escherichia coli and DNA was purified. Then, antibodies were produced by transient transfection of ExpiCHO cells according to the manufacturer's protocol (Gibco). Supernatants were filter-sterilized using $0.45-\mu \mathrm{m}$ pore size filters and samples were applied to HiTrap MabSelect SuRe columns (Cytiva).

ELISA binding of AZD8895 mutants. The wells of 384-well microtitre plates were coated with purified recombinant SARS-CoV-2 S $6 \mathrm{P}$ protein at $4{ }^{\circ} \mathrm{C}$ overnight. Plates were blocked with $2 \%$ non-fat dry milk and $2 \%$ normal goat serum in Dulbecco's PBS containing 0.05\% Tween-20 (DPBS-T) for $1 \mathrm{~h}$. Antibodies were diluted to $10 \mu \mathrm{g} \mathrm{ml}^{-1}$ and titrated twofold 23 times in DPBS-T and added to the wells, followed by an incubation for $1 \mathrm{~h}$ at room temperature. After washing with DPBS-T, a goat anti-human IgG secondary antibody conjugated with horseradish peroxidase (1:5,000 dilution, catalogue no. 2040-05, SouthernBiotech) was added and incubated for $1 \mathrm{~h}$. Plates were then washed with DPBS-T and $3,3^{\prime}, 5,5^{\prime}$-tetramethylbenzidine substrate was added to each well (Thermo Fischer Scientific). Reactions were quenched with $1 \mathrm{M}$ of hydrochloric acid and absorbance was measured at $450 \mathrm{~nm}$ using a spectrophotometer (Biotek)

\section{Mapping of mutations that escaped antibody binding. All mutations that} escaped antibody binding were mapped via a DMS approach ${ }^{29}$. We used previously described yeast display RBD mutant libraries ${ }^{29,30}$. Briefly, duplicate mutant libraries were constructed in the S RBD from SARS-CoV-2 (isolate Wuhan-Hu-1, GenBank accession no. MN908947, residues N331-T531) and contained 3,804 of the 3,819 possible amino acid mutations with $>95 \%$ present as single mutants. Each RBD variant was linked to a unique 16-nucleotide barcode sequence to facilitate downstream sequencing. As described previously, libraries were sorted for RBD expression and ACE2 binding to eliminate RBD variants that were completely misfolded or non-functional (that is, lacking modest ACE2 binding affinity ${ }^{29}$ ).

Antibody escape mapping experiments were performed in biological duplicate using two independent mutant RBD libraries, as described previously ${ }^{29}$, with minor modifications. Briefly, mutant yeast libraries induced to express RBD were washed and incubated with antibody at $400 \mathrm{ng} \mathrm{ml}^{-1}$ for $1 \mathrm{~h}$ at room temperature with gentle agitation. After antibody incubation, libraries were secondarily labelled with 1:100 fluorescein isothiocyanate-conjugated anti-MYC antibody (catalogue no. CYMC-45F; Immunology Consultants Lab) to label for RBD expression and 1:200 phycoerythrin-conjugated goat anti-human-IgG (catalogue no. 109-115098, Jackson ImmunoResearch) to label for bound antibody. Flow cytometry sorting was used to enrich for cells expressing RBD variants with reduced antibody binding via a selection gate drawn to capture unmutated SARS-CoV-2 cells labelled at $1 \%$ the antibody concentration of the library samples. For each sample, approximately 10 million $\mathrm{RBD}^{+}$cells were processed on the cytometer. Antibody-escaped cells were grown overnight in SD-CAA $\left(6.7 \mathrm{~g}^{-1}\right.$ of yeast nitrogen base, $5.0 \mathrm{gl}^{-1}$ of Casamino acid, $1.065 \mathrm{gl}^{-1}$ of 2 -( $\mathrm{N}$-morpholino)ethanesulfonic acid and $2 \% \mathrm{w} / \mathrm{v}$ dextrose) to expand cells before plasmid extraction.

Plasmid samples were prepared from preselection and overnight cultures of antibody-escaped cells (Zymoprep Yeast Plasmid Miniprep II; Zymo Research) as described previously ${ }^{29}$. The 16-nucleotide barcode sequences identifying each RBD variant were amplified by PCR and sequenced on an Illumina HiSeq 2500 with 50 base pair single-end reads as described elsewhere ${ }^{29,30}$.

Escape fractions were computed as described elsewhere ${ }^{29}$, with minor modifications as noted below. We used the dms_variants package (https:// jbloomlab.github.io/dms_variants/) v.0.8.2 to process Illumina sequences into counts of each barcoded RBD variant in each presort and antibody escape population using the barcode/RBD look-up table described previously ${ }^{74}$.

For each antibody selection, we computed the 'escape fraction' for each barcoded variant using the deep sequencing counts for each variant in the original and antibody escape populations and the total fraction of the library that escaped antibody binding via a formula described previously ${ }^{29}$. These escape fractions represent the estimated fraction of cells expressing that specific variant that fall in the antibody escape bin, such that a value of 0 means that the variant is always bound by serum and a value of 1 means that it always escapes antibody binding. We then applied a computational filter to remove variants with low sequencing counts or highly deleterious mutations that might cause antibody escape simply by leading to poor expression of properly folded RBD on the yeast cell surface ${ }^{29,30}$. Specifically, we removed variants that had (or contained mutations with) ACE2 binding scores $\leq 2.35$ or expression scores $\leq 1$, using the variant- and mutation-level DMS scores, as described previously ${ }^{30}$. Note that these filtering criteria are slightly more stringent than those previously used to map a panel of human antibodies ${ }^{29}$ but are identical to those used in recent studies defining RBD residues that impact the binding of $\mathrm{mAbs}^{74}$ and polyclonal serum ${ }^{45}$.

We next deconvolved variant-level escape scores into escape fraction estimates for single mutations using global epistasis models ${ }^{75}$ implemented in the dms_variants package, as detailed at https://jbloomlab.github.io/dms_variants/ dms_variants.globalepistasis.html and described elsewhwere ${ }^{29}$. The reported escape fractions throughout the paper are the average across the libraries (correlations shown in Extended Data Fig. 7a,b); these scores are also shown in Supplementary Table 2. Sites of strong escape from each antibody for highlighting in logo plots were determined heuristically as sites whose summed mutational escape scores were at least ten times the median sitewise sum of selection and within tenfold of the sitewise sum of the most strongly selected site. Full documentation of the computational analysis can be found at https://github.com/jbloomlab/SARS-CoV2-RBD_MAP_AZ_Abs. These results are also available in interactive format at https://jbloomlab.github.io/SARS-CoV-2-RBD_MAP_AZ_Abs/.

For plotting and analyses that required identifying RBD sites of 'strong escape' (for example, choosing which sites to show in the logo plots in Fig. 4a,b), we considered a site to mediate strong escape if the total escape (sum of mutation-level escape fractions) for that site exceeded the median across sites by greater than fivefold and was at least $5 \%$ of the maximum for any site. A markdown rendering of the identification of these sites of strong escape can be found at https://github. com/jbloomlab/SARS-CoV-2-RBD_MAP_AZ_Abs/blob/main/results/summary/ call_strong_escape_sites.md.

Antibody escape selection experiments with VSV-SARS-CoV-2. For the escape selection experiments with AZD8895 and AZD1061, we used a replication-competent recombinant VSV virus encoding the $S$ glycoprotein from SARS-CoV-2 with a 21-amino acid C-terminal deletion ${ }^{31}$. The S-expressing VSV virus was propagated in MA104 cells (African green monkey; catalogue no. CRL-2378.1; ATCC) as described previously ${ }^{31}$; viral stocks were titrated on Vero E6 cell monolayer cultures. Plaques were visualized using neutral red staining. To screen for escape mutations selected in the presence of AZD8895, AZD1061 or AZD7442, we used a real-time cell analysis assay (RTCA) and xCELLigence RTCA MP Analyzer (ACEA Biosciences) and a previously described escape selection scheme ${ }^{29}$. Briefly, $50 \mu \mathrm{l}$ of cell culture medium (DMEM supplemented with $2 \%$ FCS) was added to each well of a 96 -well E-plate to obtain a background reading. Eighteen thousand Vero E6 cells in $50 \mu \mathrm{l}$ of cell culture medium were seeded per well and plates were placed on the analyser. Measurements were taken automatically every $15 \mathrm{~min}$ and the sensograms were visualized using the RTCA software v.2.1.0 (ACEA Biosciences). VSV-SARS-CoV-2 virus (5,000 PFUs per well, approximately 0.3 multiplicity of infection) was mixed with a saturating neutralizing concentration of AZD8895, AZD1061 or AZD7442 antibody ( $5 \mu \mathrm{g} \mathrm{ml}^{-1}$ total concentration of antibodies) in a total volume of $100 \mu \mathrm{l}$ and incubated for $1 \mathrm{~h}$ at $37^{\circ} \mathrm{C}$. At $16-20 \mathrm{~h}$ after seeding the cells, the virus-antibody mixtures were added to the cell monolayers. Wells containing only virus in the absence of antibody and wells containing only Vero E6 cells in medium were included on each plate as controls. Plates were measured continuously (every $15 \mathrm{~min}$ ) for $72 \mathrm{~h}$. Escape mutations were identified by monitoring the cell index for a drop in cellular viability. To verify escape from antibody selection, wells where a cytopathic effect was observed in the presence of AZD1061 were assessed in a subsequent RTCA experiment in the presence of $10 \mu \mathrm{g} \mathrm{ml}^{-1}$ of AZD1061 or AZD8895. After confirmation of resistance of selected viruses to neutralization by AZD1061, viral isolates were expanded on Vero E6 cells in the presence of $10 \mu \mathrm{g} \mathrm{ml}^{-1}$ of AZD1061. Viral RNA was isolated using a QIAamp Viral RNA extraction kit (QIAGEN) according to the manufacturer's protocol and the SARS-CoV-2 S-gene was reverse-transcribed and amplified with a SuperScript IV One-Step RT-PCR kit (Thermo Fisher Scientific) using primers flanking the S-gene (forward: 5' -AGCTTCTGAACAATCCCCGG-3', reverse: $5^{\prime}$-GAGGCCTCTTTGAGCATGGT-3'). The amplified PCR product was purified using SPRI magnetic beads (Beckman Coulter) at a 1:1 ratio and sequenced using the Sanger method with primers giving forward and reverse reads of the RBD.

Serial passaging and testing of SARS-CoV-2 to select for mAb-resistant mutations. The SARS-CoV-2 strain USA-WA1/2020 was passaged serially in Vero cell monolayer cultures with AZD8895, AZD1061 or AZD7442 at concentrations beginning at their respective $\mathrm{IC}_{50}$ values and increased stepwise to their $\mathrm{IC}_{90}$ value with each passage. As a control, virus was passaged in the absence of antibody. After the final passage, viruses were evaluated for susceptibility to neutralization by AZD8895, AZD1061 or AZD7442, at a final concentration of $10 \times$ the $\mathrm{IC}_{90}$ concentration by plaque assay. Plaques $(n=6)$ were selected randomly for AZD1061 cultures and their virus S-encoding gene was sequenced.

The escape phenotype of selected plaque progeny viruses was validated by testing each for the six plaque-purified progeny viruses for neutralization in a plaque reduction neutralization test in comparison with the parent virus, SARS-CoV-2 (strain WA-1). All plaque-purified viruses resulted from the same $\mathrm{mAb}$ passage.

The escape phenotype of the six selected plaque progeny viruses was validated by demonstration of a loss-of-binding phenotype in biochemical tests with variant proteins incorporating selected mutations. S trimer variants were cloned into a 
HexaPro ectodomain soluble trimer construct ${ }^{76}$ with the D614G mutation and then expressed in FreeStyle 293-F cells (Thermo Fisher Scientific). Expression supernatants were collected $5 \mathrm{~d}$ posttransfection and buffer-exchanged into $1 \times$ PBS and then diluted tenfold into assay buffer ( $1 \times$ HBS-EP + buffer (Cytiva)). $R^{2}$ values indicating goodness of fit for AZD8895 or AZD1061 kinetic binding values to the $S$ trimer variants were calculated from binding traces. Relative fold shifts in $K_{D}$ were calculated in comparison to binding to WT S glycoprotein.

Generation of authentic SARS-CoV-2 viruses, including viruses with variant residues. The 2019n-CoV/USA_WA1/2020 isolate of SARS-CoV-2 was obtained from the U.S. Centers for Disease Control and passaged on Vero E6 cells. The B.1.429 isolate was generously provided by R. Andino and C. Chiu (University of California, San Francisco) and passaged once on Vero-TMPRRS2 cells. B. Pinsky (Stanford) and M. Suthar (Emory) provided the B.1.617.1 variant and R. Webby (St. Jude Children's Research Hospital) provided the B.1.617.2 variant. Individual point mutations in the S-gene (D614G and E484K/D614G) were introduced into an infectious cDNA clone of the 2019n-CoV/USA_WA1/2020 strain as described previously ${ }^{77}$. Chimeric viruses expressing the S-genes of B.1.351 or B.1.1.28 were generated in the WA1/2020 backbone and have been described previously ${ }^{42}$. Nucleotide or S-gene substitutions were introduced into an existing WA1/2020 infectious cDNA clone and assembled by in vitro ligation of seven contiguous cDNA fragments according to the protocol described previously ${ }^{78}$. In vitro transcription was performed to synthesize full-length genomic RNA. To recover the mutant or chimeric viruses, the RNA transcripts were electroporated into Vero E6 cells. The viruses from the supernatant of cells were collected $40 \mathrm{~h}$ later and served as 0 stocks. All virus stocks were confirmed by next-generation sequencing.

Focus reduction neutralization test. Serial dilutions of mAbs or serum were incubated with $10^{2}$ focus-forming units of different strains or variants of SARS-CoV-2 for $1 \mathrm{~h}$ at $37^{\circ} \mathrm{C}$. Antibody-virus complexes were added to Vero-hACE2-TMPRSS2 cell monolayer cultures in 96-well plates and incubated at $37^{\circ} \mathrm{C}$ for $1 \mathrm{~h}$. Subsequently, cells were overlaid with $1 \%(\mathrm{w} / \mathrm{v})$ methylcellulose in MEM supplemented with $2 \%$ FCS. Plates were collected $20 \mathrm{~h}$ later by removing the overlays and fixed with $4 \%$ paraformaldehyde in PBS for $20 \mathrm{~min}$ at room temperature. Plates were washed and incubated sequentially with an oligoclonal pool of anti-S murine $\mathrm{mAbs}^{44}$ and horseradish peroxidase-conjugated goat anti-human IgG in PBS supplemented with $0.1 \%$ saponin and $0.1 \%$ bovine serum albumin. SARS-CoV-2-infected cell foci were visualized using the KPL TrueBlue Peroxidase Substrate and quantitated on an ImmunoSpot microanalyser (Cellular Technologies).

Multiple sequence alignments. We searched for antibody variable gene sequences originating with the same features as those encoding AZD8895 and retrieved the matching sequences from the repertoires of each individual examined. We searched for similar sequences in the publicly available large-scale antibody sequence repertoires for three healthy individuals and cord blood repertoires (deposited with the Short Read Archive under accession no. SRP174305). The search parameters for the heavy chain were sequences with IGHV1-58 and IGHJ3 with the P99, D108 and F110 residues. Additionally, the search parameters for the light chain were sequences with the Y92 and W98 residues. Sequences from a matching clonotype that belonged to each individual were aligned with either ClustalO version 1.2.4 (heavy chains) or with MUSCLE version 3.8.31 ${ }^{80}$ (light chains). Then, logo plots of the aligned sequences were generated using WebLogo version 2.8.2 ${ }^{81}$.

Materials availability. Requests for reagents may be directed to and fulfilled by the lead contact (J.E.C.): james.crowe@vumc.org. The materials reported in this study will be made available but may require execution of a material transfer agreement.

Reporting Summary. Further information on research design is available in the Nature Research Reporting Summary linked to this article.

\section{Data availability}

The crystal structures reported in this paper have been deposited with the Protein Data Bank (https://www.rcsb.org) under accession nos. 7L7D (AZD8895 + RBD) and 7L7E (AZD8895 and AZD1061 + RBD). The following were obtained from the PDB and used for visualization or molecular replacement: 7K4N, 6M0J, 6XM4, 7CAK, 6ZOY, 6XC2 and 5JRP. The FASTQ files for DMS are available on the National Center for Biotechnology Information Sequence Read Archive under BioSample SAMN17532001 as part of BioProject PRJNA639956. Per-mutation escape fractions are available on GitHub (https://github.com/jbloomlab/ SARS-CoV-2-RBD_MAP_AZ_Abs/blob/main/results/supp_data/AZ_cocktail raw_data.csv) and in Supplementary Data Table 2. All other data are available in the main text or supplementary materials. Source data are provided with this paper.

\section{Code availability}

The computational pipeline for the DMS analysis of antibody escape mutations is available on GitHub: https://github.com/jbloomlab/ SARS-CoV-2-RBD_MAP_AZ_Abs.
Received: 2 July 2021; Accepted: 1 September 2021;

Published online: 21 September 2021

\section{References}

1. Hoffmann, M. et al. SARS-CoV-2 cell entry depends on ACE2 and TMPRSS2 and is blocked by a clinically proven protease inhibitor. Cell 181, 271-280.e8 (2020).

2. Letko, M., Marzi, A. \& Munster, V. Functional assessment of cell entry and receptor usage for SARS-CoV-2 and other lineage B betacoronaviruses. Nat. Microbiol. 5, 562-569 (2020).

3. Wahba, L. et al. An extensive meta-metagenomic search identifies SARS-CoV-2-homologous sequences in pangolin lung viromes. mSphere 5 , e00160-20 (2020).

4. Walls, A. C. et al. Tectonic conformational changes of a coronavirus spike glycoprotein promote membrane fusion. Proc. Natl Acad. Sci. USA 114, 11157-11162 (2017).

5. Algaissi, A. et al. SARS-CoV-2 S1 and N-based serological assays reveal rapid seroconversion and induction of specific antibody response in COVID-19 patients. Sci. Rep. 10, 16561 (2020).

6. Long, Q.-X. et al. Antibody responses to SARS-CoV-2 in patients with COVID-19. Nat. Med. 26, 845-848 (2020).

7. Piccoli, L. et al. Mapping neutralizing and immunodominant sites on the SARS-CoV-2 spike receptor-binding domain by structure-guided high-resolution serology. Cell 183, 1024-1042.e21 (2020).

8. Brouwer, P. J. M. et al. Potent neutralizing antibodies from COVID-19 patients define multiple targets of vulnerability. Science 369, 643-650 (2020).

9. Cao, Y. et al. Potent neutralizing antibodies against SARS-CoV-2 identified by high-throughput single-cell sequencing of convalescent patients' B cells. Cell 182, 73-84.e16 (2020)

10. Hansen, J. et al. Studies in humanized mice and convalescent humans yield a SARS-CoV-2 antibody cocktail. Science 369, 1010-1014 (2020).

11. Ju, B. et al. Human neutralizing antibodies elicited by SARS-CoV-2 infection. Nature 584, 115-119 (2020).

12. Liu, L. et al. Potent neutralizing antibodies against multiple epitopes on SARS-CoV-2 spike. Nature 584, 450-456 (2020).

13. Robbiani, D. F. et al. Convergent antibody responses to SARS-CoV-2 in convalescent individuals. Nature 584, 437-442 (2020).

14. Rogers, T. F. et al. Isolation of potent SARS-CoV-2 neutralizing antibodies and protection from disease in a small animal model. Science 369, 956-963 (2020)

15. Shi, R. et al. A human neutralizing antibody targets the receptor-binding site of SARS-CoV-2. Nature 584, 120-124 (2020)

16. Weitkamp, J.-H. et al. Infant and adult human B cell responses to rotavirus share common immunodominant variable gene repertoires. J. Immunol. 171, 4680-4688 (2003)

17. Zost, S. J. et al. Potently neutralizing and protective human antibodies against SARS-CoV-2. Nature 584, 443-449 (2020)

18. Benton, D. J. et al. Receptor binding and priming of the spike protein of SARS-CoV-2 for membrane fusion. Nature 588, 327-330 (2020)

19. Wrapp, D. et al. Cryo-EM structure of the 2019-nCoV spike in the prefusion conformation. Science 367, 1260-1263 (2020).

20. Wrobel, A. G. et al. SARS-CoV-2 and bat RaTG13 spike glycoprotein structures inform on virus evolution and furin-cleavage effects. Nat. Struct. Mol. Biol. 27, 763-767 (2020).

21. Zost, S. J. et al. Rapid isolation and profiling of a diverse panel of human monoclonal antibodies targeting the SARS-CoV-2 spike protein. Nat. Med. 26, 1422-1427 (2020)

22. Greaney, A. J. et al. Mapping mutations to the SARS-CoV-2 RBD that escape binding by different classes of antibodies. Nat. Commun. 12, 4196 (2021)

23. Tortorici, M. A. et al. Ultrapotent human antibodies protect against SARS-CoV-2 challenge via multiple mechanisms. Science $\mathbf{3 7 0}$, 950-957 (2020).

24. Kreer, C. et al. Longitudinal isolation of potent near-germline SARS-CoV-2-neutralizing antibodies from COVID-19 patients. Cell 182, 843-854.e12 (2020).

25. Farooq, A. Structuropedeida WebInterface to MODELLER. http://www. farooqed.com $/ \mathrm{mod} /$ (2021).

26. Bohne-Lang, A. \& von der Lieth, C.-W. GlyProt: in silico glycosylation of proteins. Nucleic Acids Res. 33, W214-W219 (2005).

27. Dejnirattisai, W. et al. The antigenic anatomy of SARS-CoV-2 receptor binding domain. Cell 184, 2183-2200.e22 (2021).

28. Soto, C. et al. High frequency of shared clonotypes in human B cell receptor repertoires. Nature 566, 398-402 (2019).

29. Greaney, A. J. et al. Complete mapping of mutations to the SARS-CoV-2 spike receptor-binding domain that escape antibody recognition. Cell Host Microbe 29, 44-57.e9 (2021) 
30. Starr, T. N. et al. Deep mutational scanning of SARS-CoV-2 receptor binding domain reveals constraints on folding and ACE2 binding. Cell 182, 1295-1310.e20 (2020).

31. Case, J. B. et al. Neutralizing antibody and soluble ACE2 inhibition of a replication-competent VSV-SARS-CoV-2 and a clinical isolate of SARS-CoV-2. Cell Host Microbe 28, 475-485.e5 (2020).

32. Klimstra, W. B. et al. SARS-CoV-2 growth, furin-cleavage-site adaptation and neutralization using serum from acutely infected hospitalized COVID-19 patients. J. Gen. Virol. 101, 1156-1169 (2020).

33. Sawatzki, K. et al. Host barriers to SARS-CoV-2 demonstrated by ferrets in a high-exposure domestic setting. Proc. Natl Acad. Sci. USA 118, e2025601118 (2021)

34. Baum, A. et al. REGN-COV2 antibodies prevent and treat SARS-CoV-2 infection in rhesus macaques and hamsters. Science 370, 1110-1115 (2020).

35. $\mathrm{Li}, \mathrm{Q}$. et al. The impact of mutations in SARS-CoV-2 spike on viral infectivity and antigenicity. Cell 182, 1284-1294.e9 (2020).

36. Galloway, S. E. et al. Emergence of SARS-CoV-2 B.1.1.7 lineage-United States, December 29, 2020-January 12, 2021. MMWR Morb. Mortal. Wkly Rep. 70, 95-99 (2021).

37. Leung, K., Shum, M. H., Leung, G. M., Lam, T. T. \& Wu, J. T. Early transmissibility assessment of the N501Y mutant strains of SARS-CoV-2 in the United Kingdom, October to November 2020. Euro Surveill. 26, 2002106 (2021).

38. Tegally, H. et al. Detection of a SARS-CoV-2 variant of concern in South Africa. Nature 592, 438-443 (2021).

39. Voloch, C. M. et al. Genomic characterization of a novel SARS-CoV-2 lineage from Rio de Janeiro, Brazil. J. Virol. 95, e00119-21 (2021).

40. Liu, J. et al. BNT162b2-elicited neutralization of B.1.617 and other SARS-CoV-2 variants. Nature 596, 273-275 (2021)

41. Deng, X. et al. Transmission, infectivity, and neutralization of a spike L452R SARS-CoV-2 variant. Cell 184, 3426-3437.e8 (2021).

42. Chen, R. E. et al. Resistance of SARS-CoV-2 variants to neutralization by monoclonal and serum-derived polyclonal antibodies. Nat. Med. 27, 717-726 (2021).

43. Weisblum, Y. et al. Escape from neutralizing antibodies by SARS-CoV-2 spike protein variants. eLife 9, e61312 (2020)

44. Liu, Z. et al. Identification of SARS-CoV-2 spike mutations that attenuate monoclonal and serum antibody neutralization. Cell Host Microbe 29 477-488.e4 (2021)

45. Greaney, A. J. et al. Comprehensive mapping of mutations in the SARS-CoV-2 receptor-binding domain that affect recognition by polyclonal human plasma antibodies. Cell Host Microbe 29, 463-476.e6 (2021).

46. Wibmer, C. K. et al. SARS-CoV-2 501Y.V2 escapes neutralization by South African COVID-19 donor plasma. Nat. Med. 27, 622-625 (2021).

47. Andreano, E. et al. SARS-CoV-2 escape from a highly neutralizing COVID-19 convalescent plasma. Proc. Natl. Acad. Sci. USA 118, e2103154118 (2021).

48. Cele, S. et al. Escape of SARS-CoV-2 501Y.V2 from neutralization by convalescent plasma. Nature 593, 142-146 (2021).

49. Wang, P. et al. Increased resistance of SARS-CoV-2 variant P.1 to antibody neutralization. Cell Host Microbe 29, 747-751.e4 (2021)

50. Wang, P. et al. Antibody resistance of SARS-CoV-2 variants B.1.351 and B.1.1.7. Nature 593, 130-135 (2021).

51. Wang, Z. et al. mRNA vaccine-elicited antibodies to SARS-CoV-2 and circulating variants. Nature 592, 616-622 (2021).

52. Zhou, P. et al. A pneumonia outbreak associated with a new coronavirus of probable bat origin. Nature 579, 270-273 (2020).

53. Tian, C. et al. Immunodominance of the $V_{H} 1-46$ antibody gene segment in the primary repertoire of human rotavirus-specific $\mathrm{B}$ cells is reduced in the memory compartment through somatic mutation of nondominant clones. J. Immunol. 180, 3279-3288 (2008).

54. Wu, X. et al. Focused evolution of HIV-1 neutralizing antibodies revealed by structures and deep sequencing. Science 333, 1593-1602 (2011).

55. Zhou, T. et al. Structural repertoire of HIV-1-neutralizing antibodies targeting the CD4 supersite in 14 donors. Cell 161, 1280-1292 (2015).

56. Huang, C.-C. et al. Structural basis of tyrosine sulfation and $\mathrm{V}_{\mathrm{H}^{-}}$-gene usage in antibodies that recognize the HIV type 1 coreceptor-binding site on gp120. Proc. Natl Acad. Sci. USA 101, 2706-2711 (2004).

57. Williams, W. B. et al. Diversion of HIV-1 vaccine-induced immunity by gp41-microbiota cross-reactive antibodies. Science 349, aab1253 (2015)

58. Joyce, M. G. et al. Vaccine-induced antibodies that neutralize group 1 and group 2 influenza A viruses. Cell 166, 609-623 (2016).

59. Pappas, L. et al. Rapid development of broadly influenza neutralizing antibodies through redundant mutations. Nature 516, 418-422 (2014)

60. Sui, J. et al. Structural and functional bases for broad-spectrum neutralization of avian and human influenza A viruses. Nat. Struct. Mol. Biol. 16, 265-273 (2009).

61. Wheatley, A. K. et al. H5N1 vaccine-elicited memory B cells are genetically constrained by the IGHV locus in the recognition of a neutralizing epitope in the hemagglutinin stem. J. Immunol. 195, 602-610 (2015).
62. Bailey, J. R. et al. Broadly neutralizing antibodies with few somatic mutations and hepatitis C virus clearance. JCI Insight 2, e92872 (2017).

63. Giang, E. et al. Human broadly neutralizing antibodies to the envelope glycoprotein complex of hepatitis C virus. Proc. Natl Acad. Sci. USA 109, 6205-6210 (2012).

64. Yuan, M. et al. Structural basis of a shared antibody response to SARS-CoV-2. Science 369, 1119-1123 (2020).

65. Nielsen, S. C. A. et al. Human B cell clonal expansion and convergent antibody responses to SARS-CoV-2. Cell Host Microbe 28, 516-525.e5 (2020).

66. Rappuoli, R., Bottomley, M. J., D’Oro, U., Finco, O. \& De Gregorio, E. Reverse vaccinology 2.0: human immunology instructs vaccine antigen design. J. Exp. Med. 213, 469-481 (2016).

67. Thomson, E. C. et al. Circulating SARS-CoV-2 spike N439K variants maintain fitness while evading antibody-mediated immunity. Cell 184, 1171-1187.e20 (2021)

68. van Dorp, L. et al. Recurrent mutations in SARS-CoV-2 genomes isolated from mink point to rapid host-adaptation. Preprint at bioRxiv https://doi. org/10.1101/2020.11.16.384743 (2020)

69. Kabsch, W. XDS. Acta Crystallogr. D Biol. Crystallogr. 66, 125-132 (2010)

70. Winn, M. D. et al. Overview of the CCP4 suite and current developments. Acta Crystallogr. D Biol. Crystallogr. 67, 235-242 (2011).

71. McCoy, A. J. et al. Phaser crystallographic software. J. Appl. Crystallogr. 40, 658-674 (2007)

72. Adams, P. D. et al. PHENIX: a comprehensive Python-based system for macromolecular structure solution. Acta Crystallogr. D Biol. Crystallogr. 66, 213-221 (2010)

73. Emsley, P. \& Cowtan, K. Coot: model-building tools for molecular graphics. Acta Crystallogr. D Biol. Crystallogr. 60, 2126-2132 (2004).

74. Starr, T. N. et al. Prospective mapping of viral mutations that escape antibodies used to treat COVID-19. Science 371, 850-854 (2021).

75. Otwinowski, J., McCandlish, D. M. \& Plotkin, J. B. Inferring the shape of global epistasis. Proc. Natl Acad. Sci. USA 115, E7550-E7558 (2018).

76. Hsieh, C.-L. et al. Structure-based design of prefusion-stabilized SARS-CoV-2 spikes. Science 369, 1501-1505 (2020).

77. Plante, J. A. Spike mutation D614G alters SARS-CoV-2 fitness. Nature 592 116-121 (2021)

78. Xie, X. et al. An infectious cDNA clone of SARS-CoV-2. Cell Host Microbe 27, 841-848.e3 (2020)

79. Sievers, F. et al. Fast, scalable generation of high-quality protein multiple sequence alignments using Clustal Omega. Mol. Syst. Biol. 7, 539 (2011).

80. Edgar, R. C. MUSCLE: a multiple sequence alignment method with reduced time and space complexity. BMC Bioinformatics 5, 113 (2004).

81. Crooks, G. E., Hon, G., Chandonia, J.-M. \& Brenner, S. E. WebLogo: a sequence logo generator. Genome Res. 14, 1188-1190 (2004).

\section{Acknowledgements}

At the Fred Hutchinson Cancer Research Center, we thank A. Addetia for experimental assistance, the Flow Cytometry and Genomics core facilities and Scientific Computing supported by an ORIP grant no. S10OD028685. We thank A. Creanga and B. Graham of the U.S. National Institutes of Health (NIH) for the Vero-hACE2-TMPRSS2 cells. At AstraZeneca, we thank P. Warrener, C. Morehouse and D. Tabor for virus genome sequencing and spike variant analysis and $\mathrm{K}$. Ren for the generation of protein reagents and related binding data. We thank B. Pinsky (Stanford) and M. Suthar (Emory) for providing the B.1.617.1 variant and R. Webby (St. Jude Children's Research Hospital) for the B.1.617.2 variant. Some schematics were created with Biorender.com. This work was supported by Defense Advanced Research Projects Agency grant nos. HR0011-18-2-000 and HR0011-18-3-0001, NIH contract nos. 75N93019C00074 and 75N93019C00062, NIH grant nos. AI150739, AI157155, AI141707, AI083203, AI095202 and UL1TR001439, the Dolly Parton COVID-19 Research Fund at Vanderbilt, a grant from Fast Grants, Mercatus Center, George Mason University and funding from AstraZeneca. T.N.S. is a Washington Research Foundation Innovation Fellow at the University of Washington Institute for Protein Design and a Howard Hughes Medical Institute Fellow of the Damon Runyon Cancer Research Foundation (no. DRG-2381-19). J.E.C. is a recipient of the 2019 Future Insight Prize from Merck, which supported this work with a grant. J.D.B. is an investigator of the Howard Hughes Medical Institute. P.-Y.S. was supported by awards from the Sealy \& Smith Foundation, Kleberg Foundation, John S. Dunn Foundation, Amon G. Carter Foundation, the Gilson Longenbaugh Foundation and the Summerfield Robert Foundation. J.B.C. is supported by a Helen Hay Whitney Foundation postdoctoral fellowship. X-ray diffraction data were collected at Beamline 21-ID-F and 21-ID-G at the Advanced Photon Source, a U.S. Department of Energy Office of Science User Facility operated for the Office of Science by Argonne National Laboratory under contract no. DE-AC02-06CH11357. Use of the LS-CAT Sector 21 was supported by the Michigan Economic Development Corporation and Michigan Technology Tri-Corridor (grant no. 085P1000817). Support for crystallography was provided from the Vanderbilt Center for Structural Biology. The content is solely the responsibility of the authors and does not necessarily represent the official views of the U.S. Government or the other sponsors. 


\section{Author contributions}

J.D., S.J.Z., J.D.B. and J.E.C. conceptualized the study. J.D., S.J.Z., A.J.G., T.N.S., A.S.D., E.C.C., R.E.C., J.B.C., R.E.S., P.G., J.R., E.A., C.G., R.S.N., E.B., X.X., X.Z., J.L., S.W. M.E.M., M.B.F., T.B., K.M.T., H.B., Y-M.L. and P.M. carried out the investigation. P.-Y.S., M.T.E., M.S.D., J.D.B. and J.E.C. supervised the study. P.-Y.S., M.T.E., R.H.C., M.S.D. J.D.B. and J.E.C. acquired the funding. J.D., S.J.Z. and J.E.C. wrote the original draft. All authors edited the manuscript and approved the final submission.

\section{Competing interests}

T.B., K.M.T., H.B., Y-M.L., P.M.M. and M.T.E. are employees of and may own stock in AstraZeneca. M.S.D. is a consultant for InBios International, Vir Biotechnology, Fortress Biotech and Carnival Corporation and on the scientific advisory boards of Moderna and Immunome. The Diamond laboratory has received funding support in sponsored research agreements from Moderna, Vir Biotechnology and Emergent BioSolutions. J.E.C. has served as a consultant for Luna Biologics, is a member of the scientific advisory board of Meissa Vaccines and is founder of IDBiologics. The Crowe laboratory at Vanderbilt University Medical Center has received sponsored research agreements from Takeda Vaccines, IDBiologics and AstraZeneca. Vanderbilt University has applied for patents concerning antibodies that are related to this work. Vanderbilt University has licensed certain rights to antibodies described in this paper to AstraZeneca. All other authors declare no competing interests.

\section{Additional information}

Extended data is available for this paper at https://doi.org/10.1038/s41564-021-00972-2.

Supplementary information The online version contains supplementary material available at https://doi.org/10.1038/s41564-021-00972-2.

Correspondence and requests for materials should be addressed to James E. Crowe.

Peer review information Nature Microbiology thanks Constantinos Kurt Wibmer and the other, anonymous, reviewers for their contribution to the peer review of this work.

Reprints and permissions information is available at www.nature.com/reprints. Publisher's note Springer Nature remains neutral with regard to jurisdictional claims in published maps and institutional affiliations.

(C) The Author(s), under exclusive licence to Springer Nature Limited 2021 


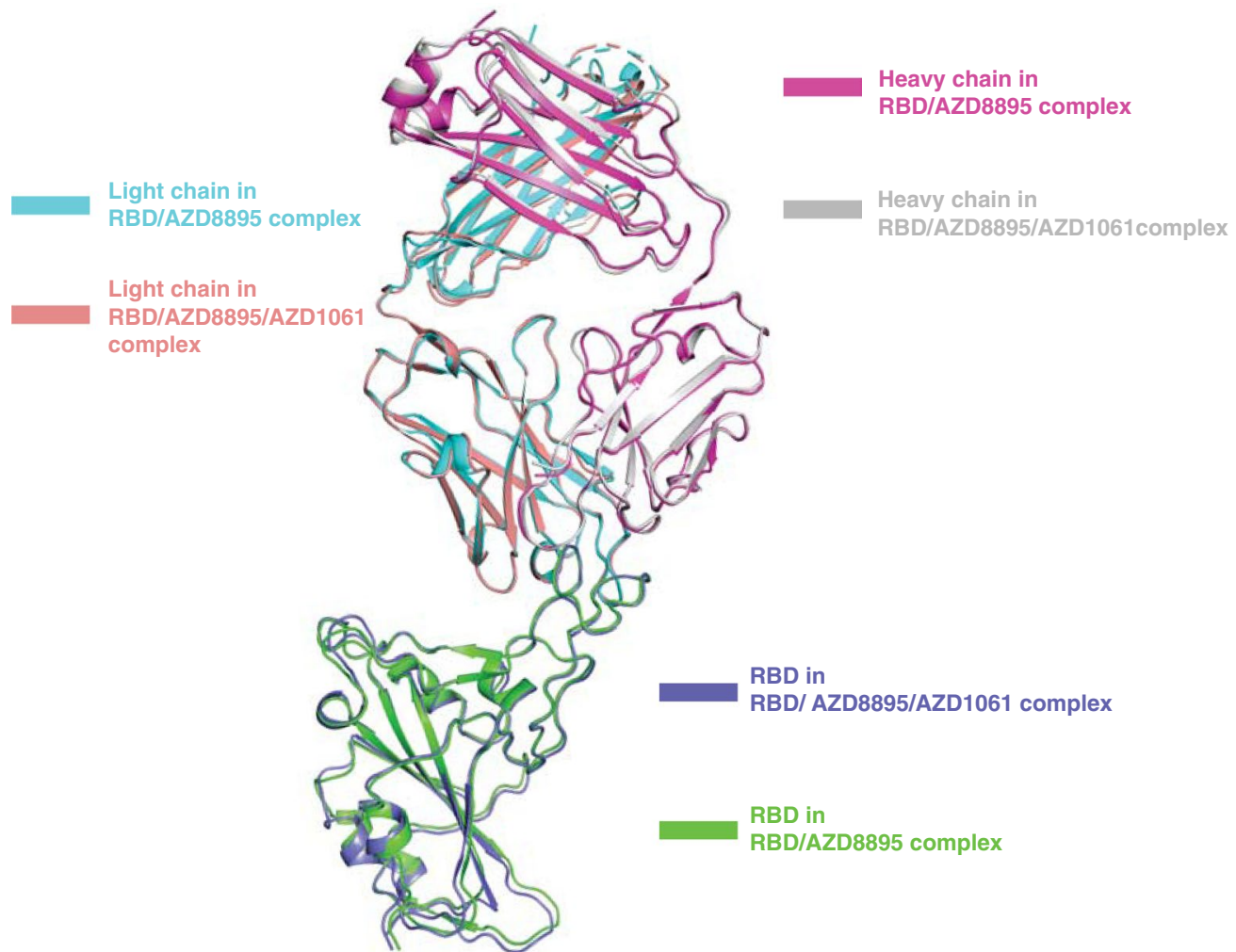

Extended Data Fig. 1 | Overlay of substructure of RBD/AZD8895 in RBD/AZD8895/AZD1061 complex and RBD/AZD8895 crystal structure. 


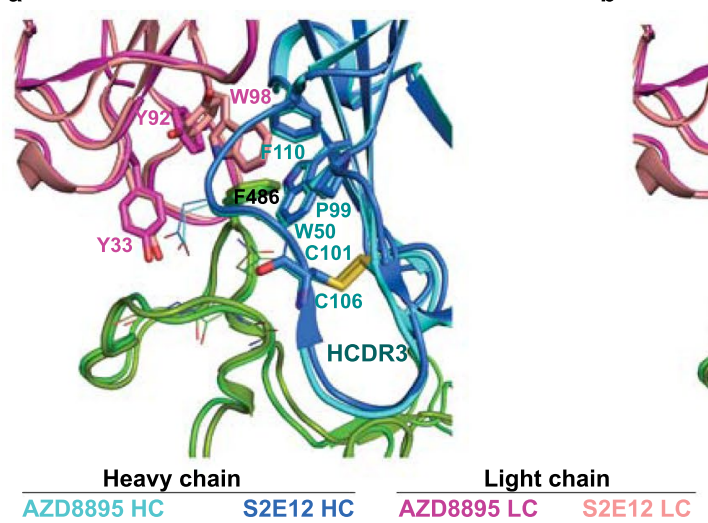

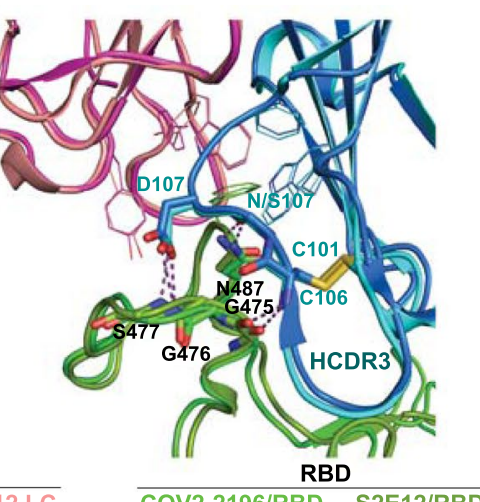

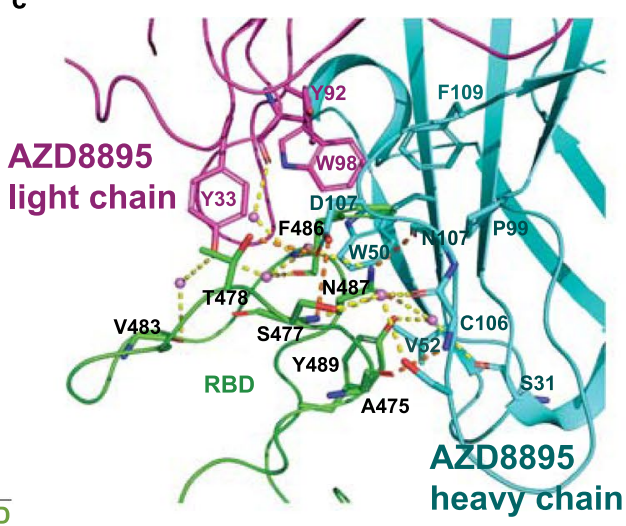

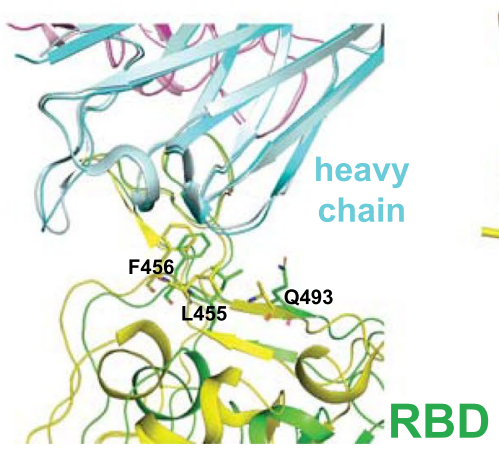

e

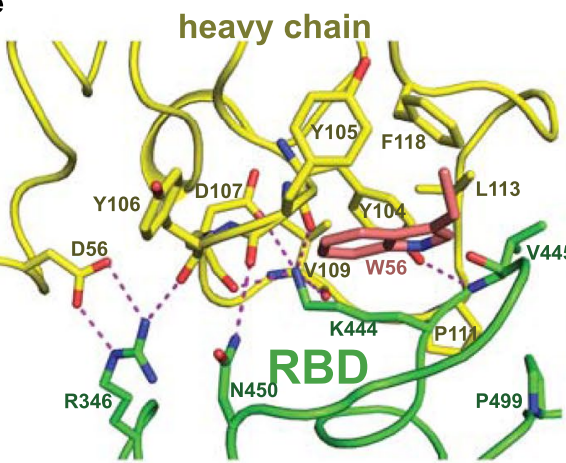

f

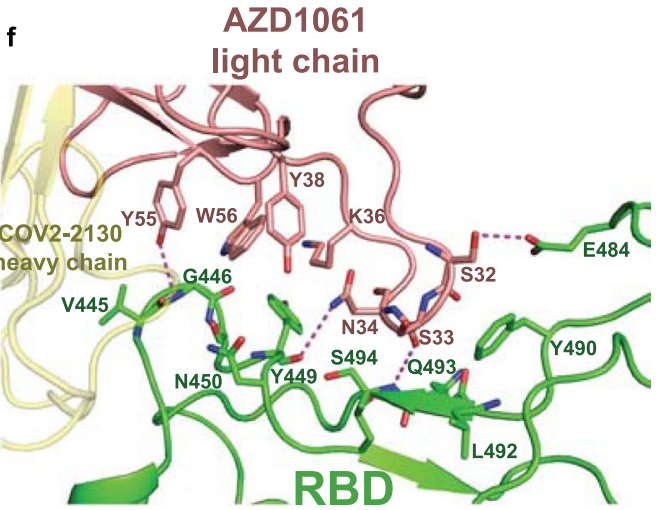

Extended Data Fig. 2 | Similarities in structural details of interactions between RBD and AZD8895, AZD1061 and those seen in the spike/S2E12 complex. a. Similar aromatic stacking and hydrophobic interaction patterns at the RBD site F486 shared between RBD/AZD8895 and spike/S2E12 complexes. b. Same hydrogen bonding pattern surrounding residue F486 in the structures of the RBD/AZD8895 and spike/S2E12 complexes. c. Detailed interactions between AZD8895 and RBD. AZD8895 heavy chain is colored in cyan, the light chain is colored in magenta, and RBD is colored in green. Important interacting residues are shown in stick representation. Water molecules involved in antibody-RBD interaction are represented as pink spheres. Direct hydrogen bonds are shown as orange dashed lines, and water-mediated hydrogen bonds as yellow dashed lines. d. Superimposition of RBD/S2E12 cryo-EM structure onto the RBD/AZD8895 crystal structure, with the variable domains of antibodies as references. AZD8895 heavy chain is in cyan, and its light chain in magenta; S2E12 heavy chain is in pale cyan, and its light chain in light pink. The two corresponding RBD structures are colored in green or yellow, respectively. e. Detailed interactions between AZD1061 heavy chain and RBD. Paratope residues are shown in stick representation and colored in yellow, epitope residues in green sticks. Hydrogen-bonds or strong polar interactions are represented as dashed magenta lines. $\mathbf{f}$. Detailed interactions between AZD1061 light chain and RBD. Paratope residues are shown in stick representation and colored in orange, epitope residues in green sticks. Hydrogen-bonds are represented as dashed magenta lines. 
a

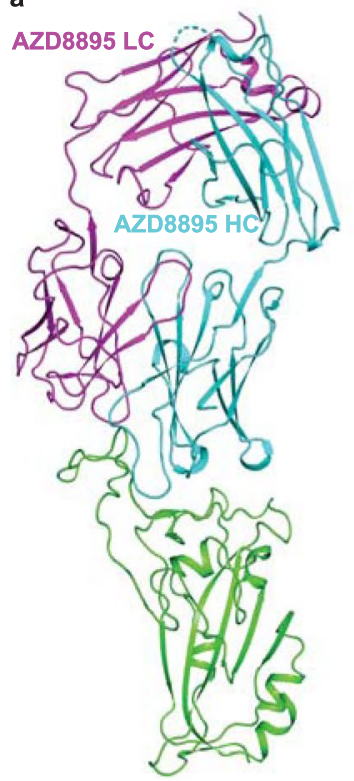

RDB/AZD8895

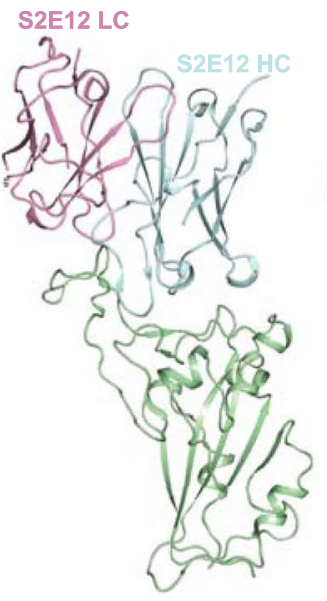

RDB/S2E12 c

COV2-2381 LC

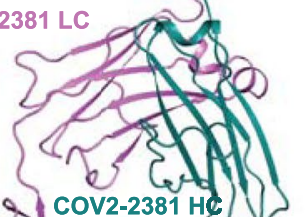
Cov2-2381 HE)

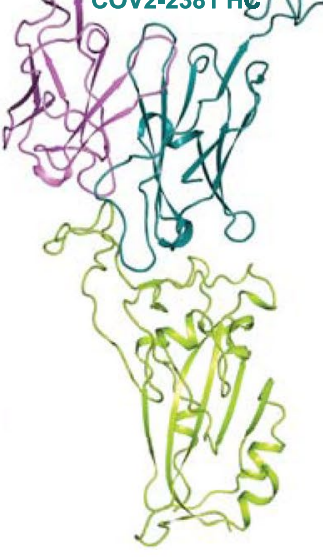

RDB/COV2-2381 d

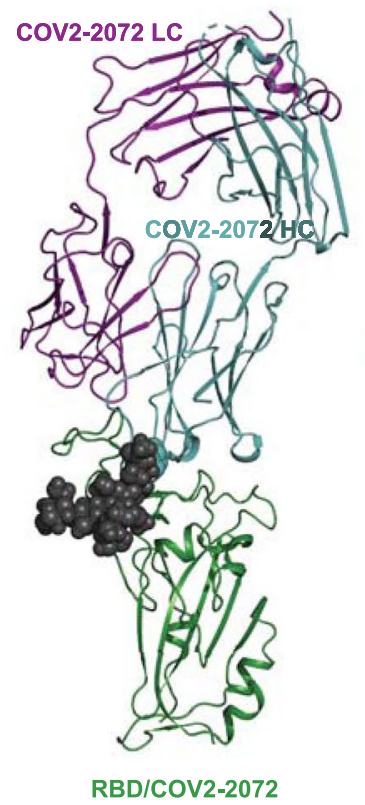

e
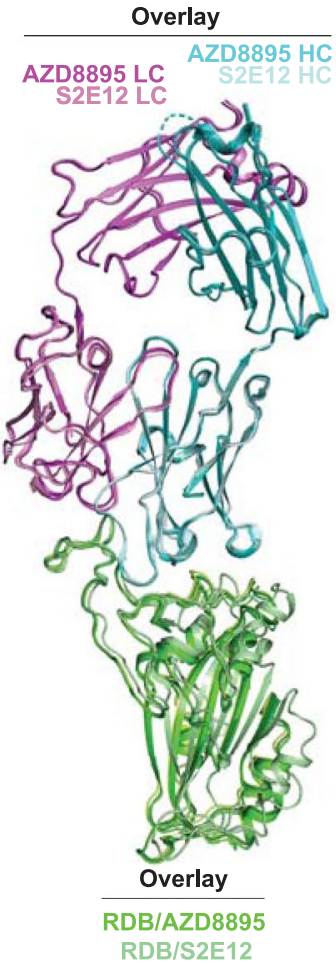

Extended Data Fig. 3 | A common clonotype of anti-RBD antibodies with the same binding mechanism. a. RBD/AZD8895 crystal structure. b. RBD/ S2E12 cryo-EM structure. c. RBD/COV2-2381 homology model generated with Structuropedia ${ }^{25}$. COV2-2072 encodes an N-linked glycosylation sequon in the HCDR3, indicated by the gray spheres, which was modeled using the GlyProt webserver. d. RBD/COV2-2072 homology model generated with Structuropedia ${ }^{25}$. e. Overlay of the RBD/AZD8895 crystal structure (a) and RBD/S2E12 cryo-EM structure (b). 


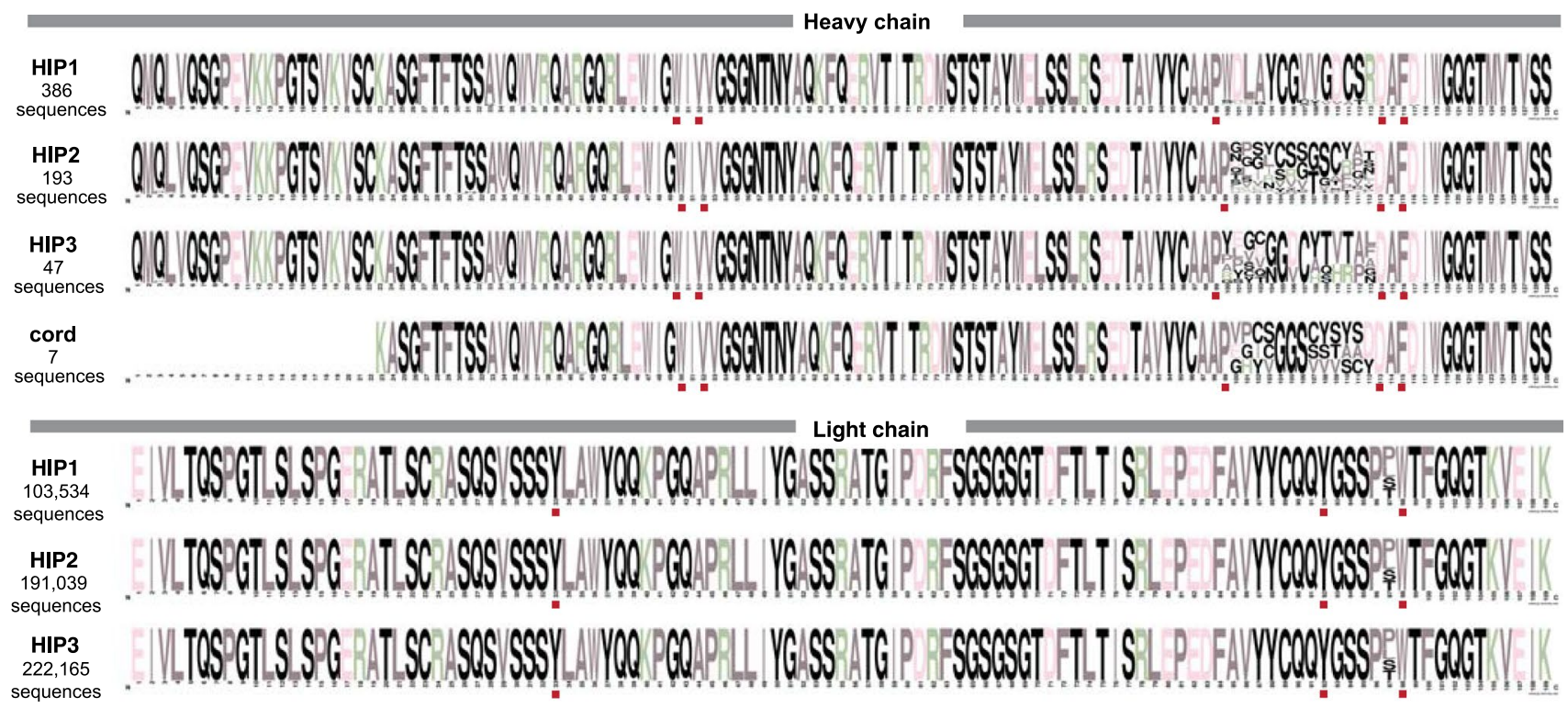

b

EIVLTQSPGTLSLSPGERATLSCRASOSVSSSYLAWYQQKPGQAPRLLIYGASSRATGIPDRFSGSGSGTDFTLTISRLEPEDFAVYYCQQYGSS-PWTFGQGTKVEIK EIVLTQSPGTLSLSPGERATLSCRASQSVSSSYLAWYQQKPGQAPRLLIYGASSRATGIPDRFSGSGSGTDFTLTISRLEPEDFAVYYCQQYGSSPPWTFGQGTKVEIK $\overline{0}$ EIVLTQSPGTLSLSPGERATLSCRASQSVSSSYLAWYQQKPGQAPRLLIYGASSRATGIPDRFSGSGSGTDFTLTISRLEPEDFAVYYCQQYGSS-LWTFGQGTKVEIK

IIVLTQSPGTLSLSPGERATLSCRASQSVSSSYLAWYQQKPGQAPRLLIYGASSRATGIPDRFSGSGSGTDFTLTISRLEPEDFAVYYCQQYGSS-SWTFGQGTKVEIK EIVLTQSPGTLSLSPGERATLSCRASQSVSSSYLAWYQQKPGQAPRLLIYGASSRATGIPDRFSGSGSGTDFTLTISRLEPEDFAVYYCQQYGSSPTWTFGQGTKVEIK

EIVLTQSPGTLSLSPGERATLSCRASQSVSSSYLAWYQQKPGQAPRLLIYGASSRATGIPDRFSGSGSGTDFTLTISRLEPEDFAVYYCQQYGSS-PWTFGQGTKVEIK N EIVLTQSPGTLSLSPGERATLSCRASQSVSSSYLAWYQQKPGQAPRLLIYGASSRATGIPDRFSGSGSGTDFTLTISRLEPEDFAVYYCQQYGSS-LWTFGQGTKVEIK 言 EIVLTQSPGTLSLSPGERATLSCRASQSVSSSYLAWYQQKPGQAPRLLIYGASSRATGIPDRFSGSGSGTDFTLTISRLEPEDFAVYYCQQYGSSPPWTFGQGTKVEIK EIVLTQSPGTLSLSPGERATLSCRASQSVSSSYLAWYQQKPGQAPRLLIYGASSRATGIPDRFSGSGSGTDFTLTISRLEPEDFAVYYCQQYGSS-SWTFGQGTKVEIK EIVLTQSPGTLSLSPGERATLSCRASQSVSSSYLAWYQQKPGQAPRLLIYGASSRATGIPDRFSGSGSGTDFTLTISRLEPEDFAVYYCQQYGSSPTWTFGQGTKVEIK

EIVLTQSPGTLSLSPGERATLSCRASQSVSSSYLAWYQQKPGQAPRLLIYGASSRATGIPDRFSGSGSGTDFTLTISRLEPEDFAVYYCQQYGSS-PWTFGQGTKVEIK EIVLTQSPGTLSLSPGERATLSCRASQSVSSSYLAWYQQKPGQAPRLLIYGASSRATGIPDRFSGSGSGTDFTLTISRLEPEDFAVYYCQQYGSS-LWTFGQGTKVEIK 产 EIVLTQSPGTLSLSPGERATLSCRASQSVSSSYLAWYQQKPGQAPRLLIYGASSRATGIPDRFSGSGSGTDFTLTISRLEPEDFAVYYCQQYGSSPPWTFGQGTKVEIK EIVLTQSPGTLSLSPGERATLSCRASQSVSSSYLAWYQQKPGQAPRLLIYGASSRATGIPDRFSGSGSGTDFTLTISRLEPEDFAVYYCQQYGSS-SWTFGQGTKVEIK EIVLTQSPGTLSLSPGERATLSCRASQSVSSSYLAWYQQKPGQAPRLLIYGASSRATGIPDRFSGSGSGTDFTLTISRLEPEDFAVYYCQQYGSSPTWTFGQGTKVEIK

Extended Data Fig. 4 | Identification of putative public clonotype members genetically similar to AZD8895 in the antibody variable gene repertoires of virus-naïve individuals. Antibody variable gene sequences collected from healthy individuals (HIP1,2, or 3) prior to the pandemic with the same sequence features as AZD8895 heavy chain and light chain are aligned. a. WebLogo plots of heavy chain (top) and light chain (bottom) sequences from three different adult donors and cord blood samples with the features of the public clonotype. The sequence features and contact residues used in AZD8895 are highlighted in red boxes below each multiple sequence alignment. b. Since the light chain plots in (a) showed restricted diversity, here we show amino acid alignments for the top five representative light chains that occurred most frequently in the three adult donors studied (HIP1, 2, or 3). 


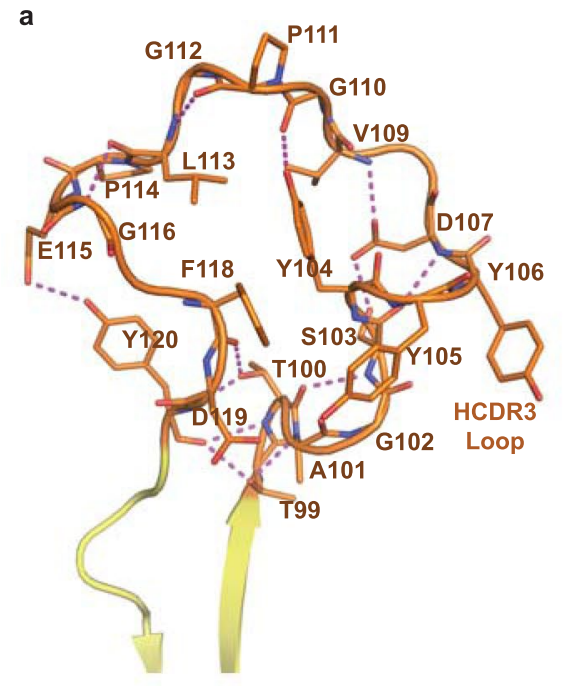

b

c
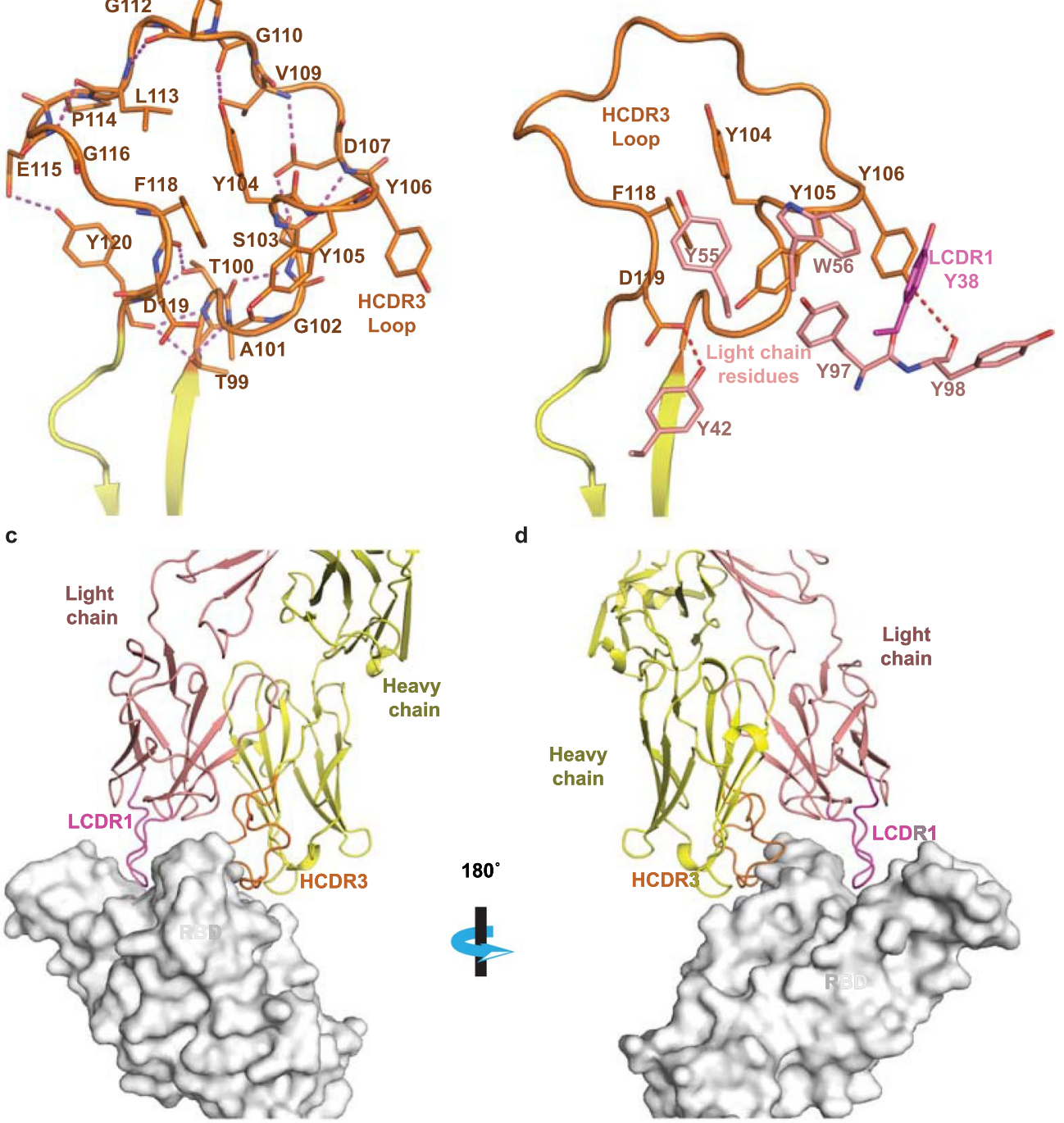

d
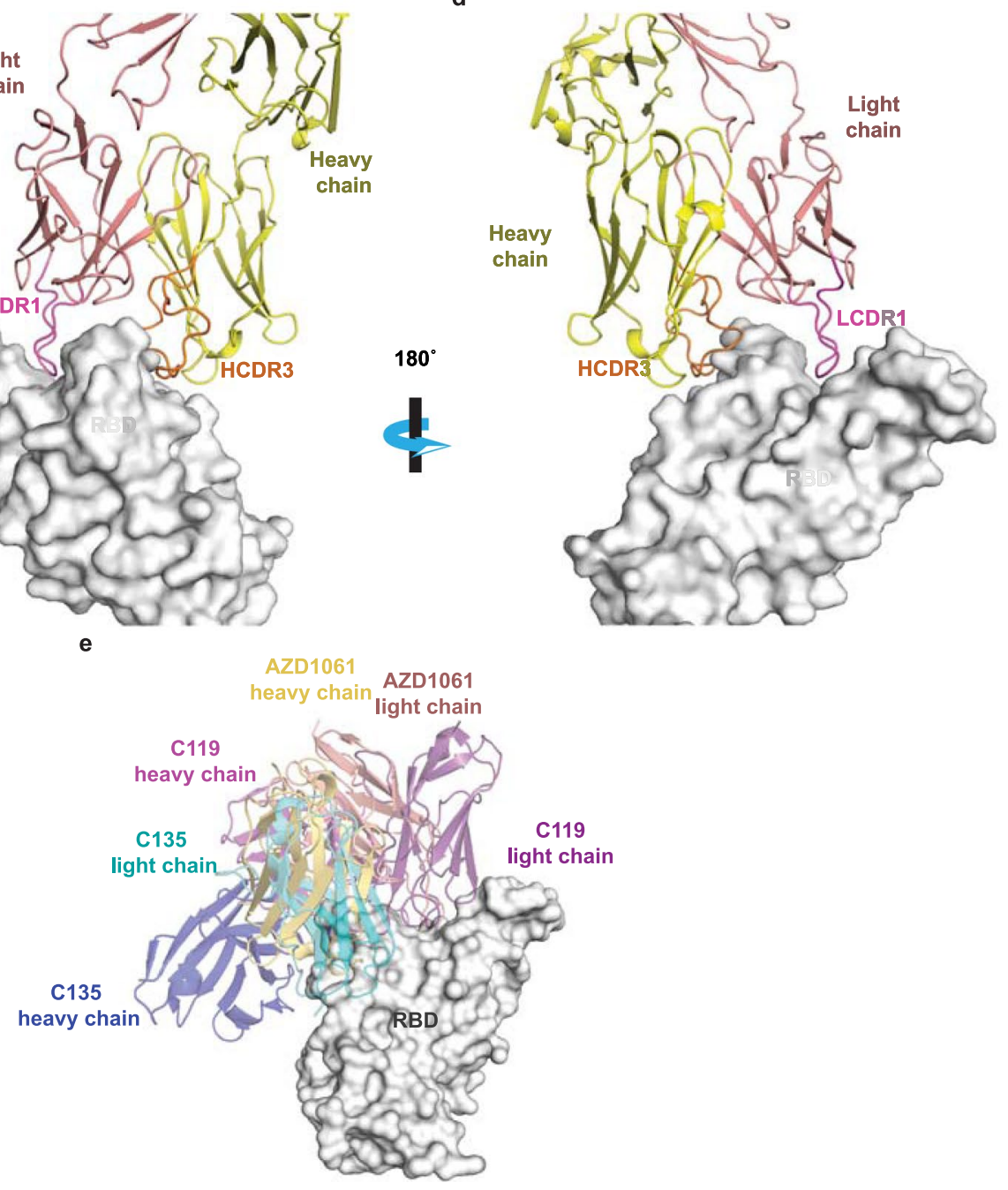

Extended Data Fig. 5 | Details of AZD1061 interaction with SARS-CoV-2 S protein RBD. a. Detailed AZD1061 HCDR3 loop structure. Short-range hydrogen bonds, stabilizing the loop conformation, are shown as dashed magenta lines. $\mathbf{b}$. Residues of AZD1061 light chain form aromatic stacking interactions and hydrogen bonds with HCDR3 to further stabilize the HCDR3 loop. LCDR1 residue Y38 is colored in magenta to match the LCDR1 coloring in panels (c) and (d). c. Long LCDR1, HCDR2, and HCDR3 form complementary binding surface to the RBD epitope. RBD is shown as surface representation in grey. AZD1061 heavy chain is colored in yellow with HCDR3 in orange, and the light chain in pink with LCDR1 in magenta. $\mathbf{d}$. $180^{\circ}$ rotation view of panel c. e. Comparison of AZD1061 binding with the previously published mAbs C119 (PDB ID: 7KHW) and C135 (PDB ID: 7K8Z). 
Interaction of Fab AZD1061 and Fab AZD8895 when bound to RBD

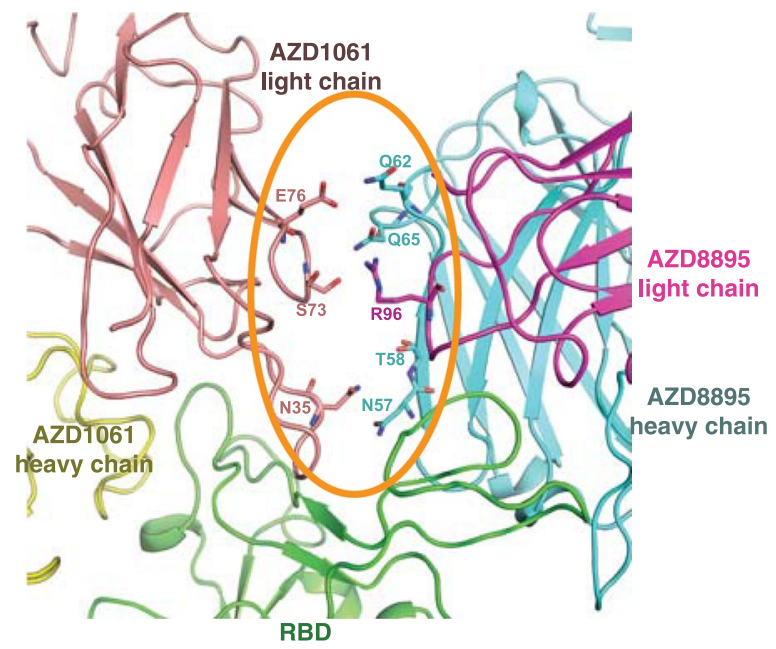

Extended Data Fig. 6 | Interface between AZD8895 and AZD1061 in the RBD/AZD8895/AZD1061 crystal structure. AZD8895 heavy or light chain are shown as cartoon representation in cyan or magenta, respectively, and AZD1061 heavy or light chains in yellow or pink, respectively. The RBD is colored in green. Interface residues are shown in stick representation. 

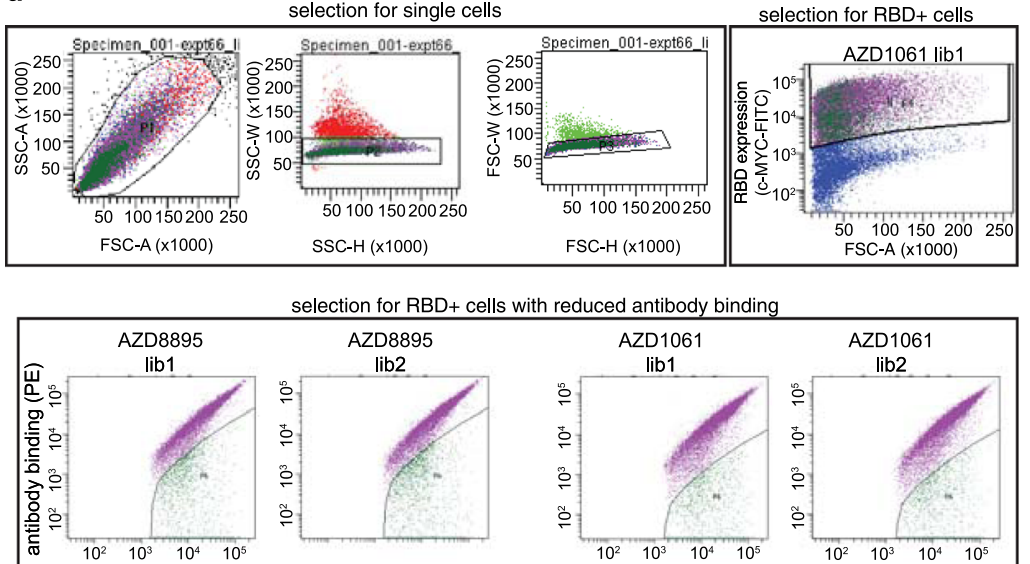

selection for $\mathrm{RBD}+$ cells with reduced antibody binding
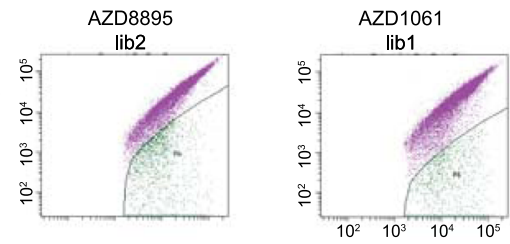

RBD expression (CMYC-FITC)

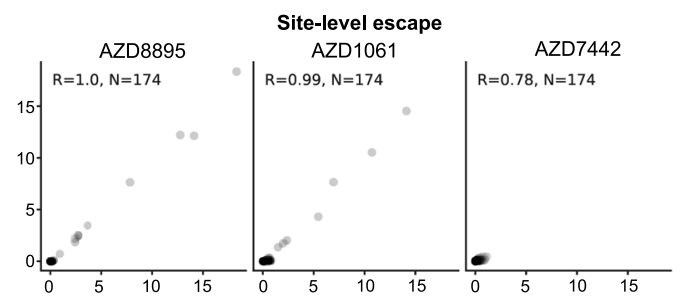

C
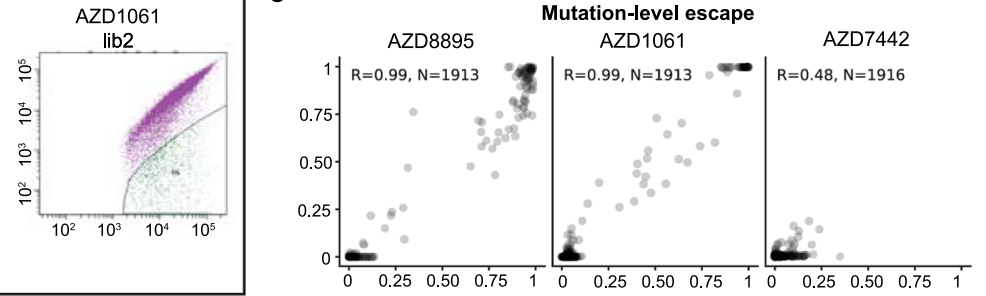

d
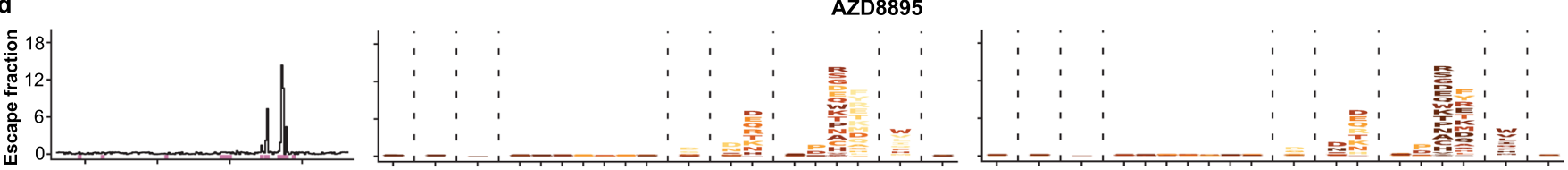

e
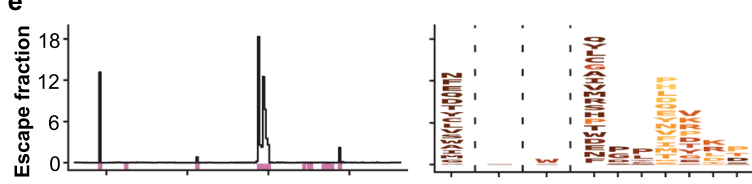

AZD1061

f

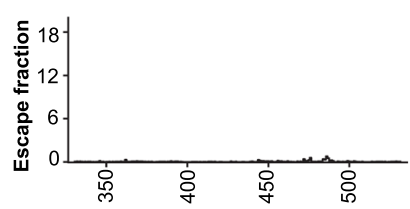

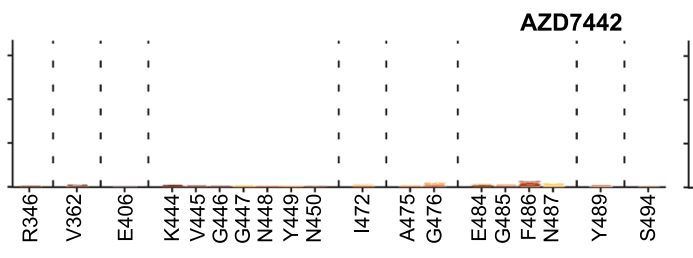

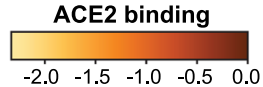

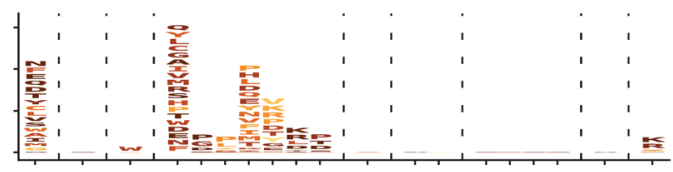

g

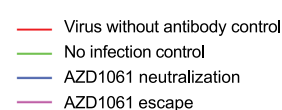

- AZD1061 neutralization

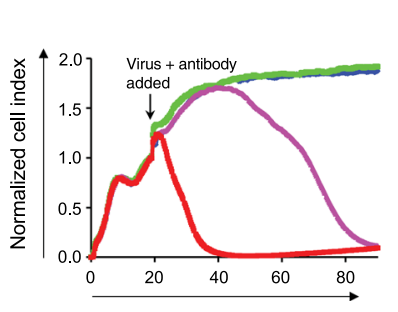

Time (h) h

_- AZD1061 escape virus (no antibody)

- No infection control

- AZD1061 escape virus + AZD8895

- AZD1061 escape virus + AZD1061

Virus + antibody

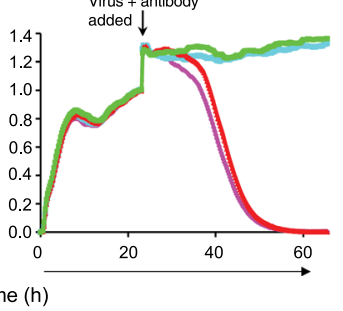

i

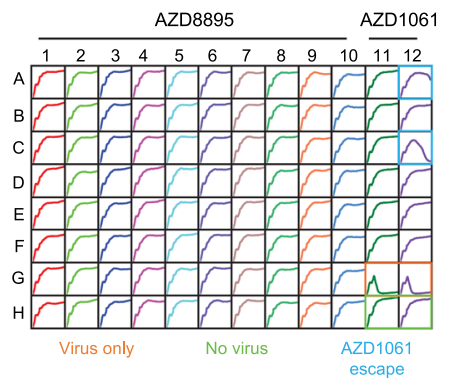

RBD expression

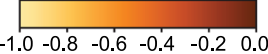

Extended Data Fig. 7 | See next page for caption. 
Extended Data Fig. 7 | Identification by deep mutational scanning of mutations affecting antibody binding and method of selection of antibody resistant mutants with VSV-SARS-CoV-2 virus. a. Top: Plots showing gating strategy for selection of single yeast cells using forward- and side-scatter (first three panels) or RBD expression (right panel). Each plot is derived from the preceding gate. Bottom: Plots showing gating for RBD ${ }^{+}$, antibody ${ }^{-}$yeast cells. Selection experiments are shown for AZD8895 or AZD1061 - two independent libraries each. b. Correlation of sites of escape between yeast library selection experiments. The $x$-axes show cumulative escape fraction for each site for library 1 , and the $y$-axes show cumulative escape fraction for each site for library 2. Correlation coefficient and $n$ are denoted for each graph. c. Correlation of observed mutations that escape antibody binding between yeast library selection experiments. The $x$-axes show each amino acid mutation's escape fraction for library 1 , and the $y$-axes show each amino acid mutation's escape fraction for library 2. Correlation coefficient and $n$ are denoted for each graph. d-f. DMS results for AZD8895 (d), AZD1061 (e), or AZD7442 (f). Left panels: sites of escape across the entire RBD indicated by peaks that correspond to the logo plots in the middle/right panels. Middle panel: logo plot of cumulative escape mutation fractions of all RBD sites with strong escape mutations. Mutations are colored based on degree to which they abrogate RBD binding to hACE2. Right: logo plots show cumulative escape fractions, but colored based on degree to which mutations affect RBD expression. g. RTCA sensograms showing neutralization escape. Cytopathic effect was monitored kinetically in cells inoculated with virus in the presence of $5 \mu \mathrm{g} / \mathrm{mL}$ AZD1061. Representative escape (magenta) or lack of escape (blue) are shown. Green - uninfected cells; red -cells inoculated with virus without antibody. Magenta/ blue curves - a single representative well; red/green controls - mean of technical duplicates. $\mathbf{h}$. Representative RTCA sensograms validating that a virus selected by AZD1061 in (g) escaped AZD1061 (magenta) but not AZD8895 (light blue). i. Example sensograms from wells of 96-well E-plate analysis for escape selection. Instances of escape from AZD1061 are noted, while escape was not detected in the presence of AZD8895 or AZD7442. Positive and negative controls are denoted on the first plate. 
Schematic for selection of SARS-CoV-2 resistant variants through serial passaging Final passage at
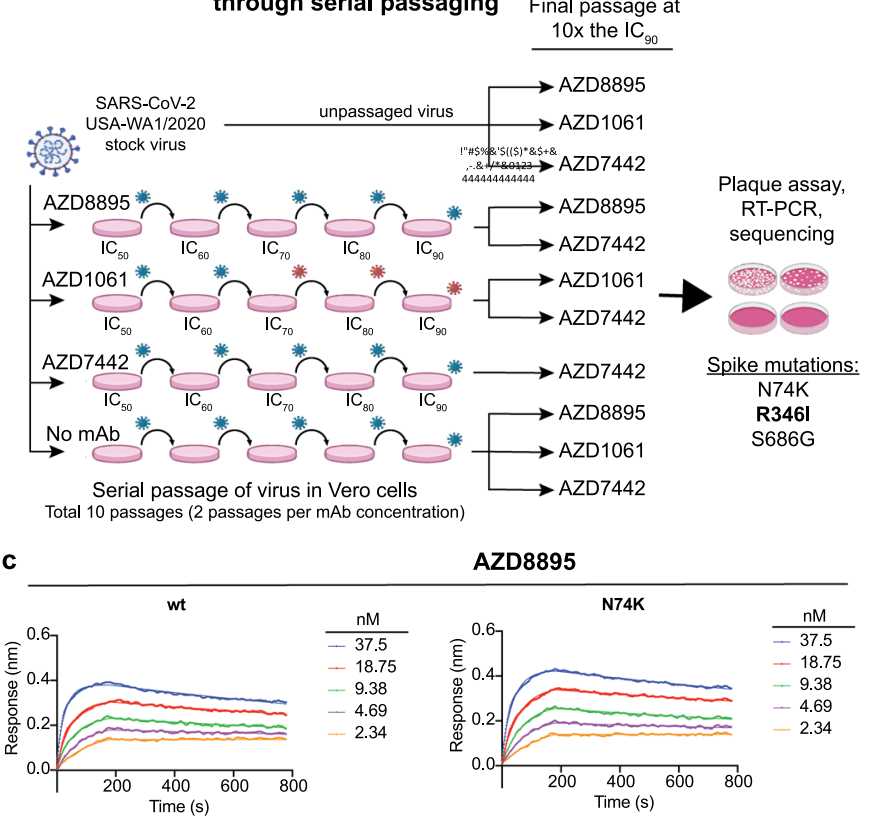

AZD8895
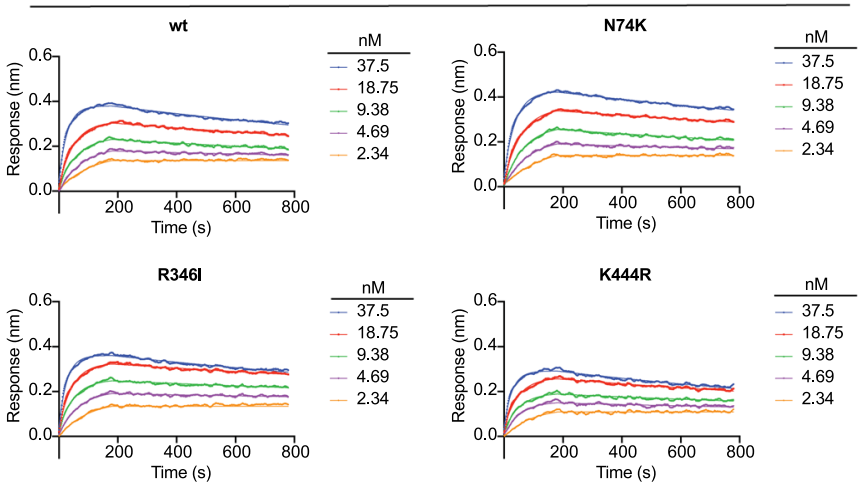

K444E
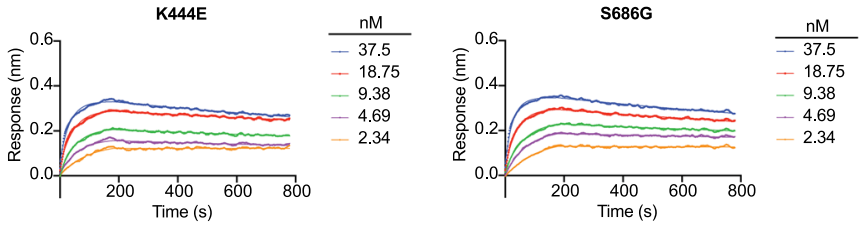

Validation of SARS-CoV-2 variant neutralization resistance (PRNT)

Treatment: AZD1061

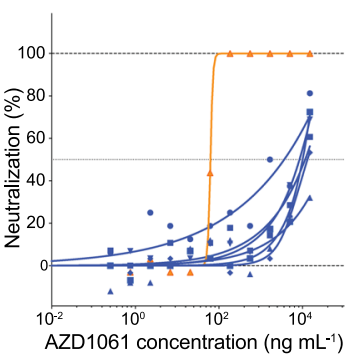

- SARS-CoV-2 (WA-1) - SARS-CoV-2 (AZD1061-Resistant)

Treatment: AZD7442

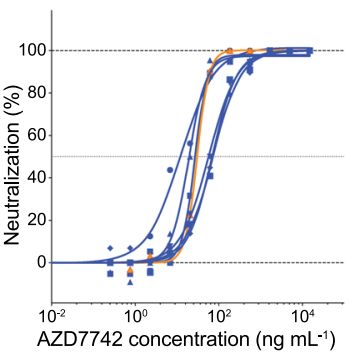

AZD1061

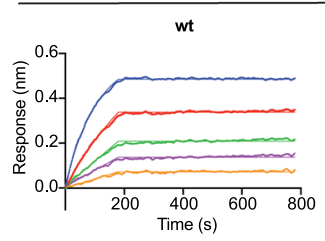

R3461
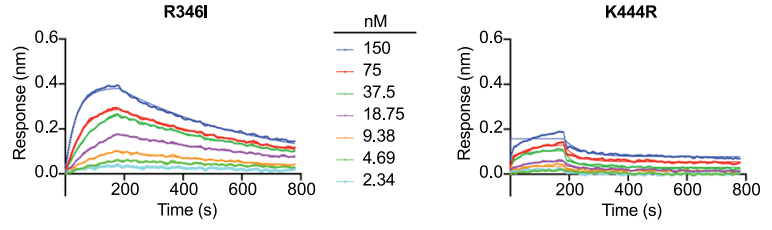

-5000
-2500
-1250
-625
-312.5
-156.25
-78.13

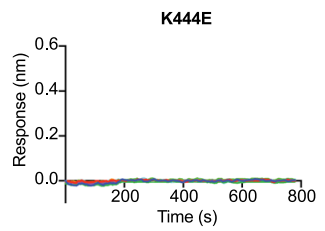

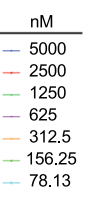

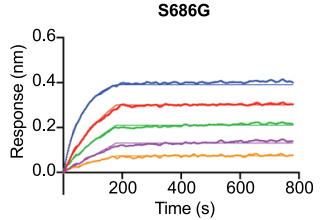

AZD8895

AZD1061

\begin{tabular}{|c|c|c|c|c|c|c|c|c|c|c|}
\hline \multirow[b]{2}{*}{$\begin{array}{c}\text { Spike } \\
\text { variant } \\
\text { (D614G S6P) }\end{array}$} & & \\
\hline & $K_{D}(M)$ & Kon (1/Ms) & $k_{\text {off }}(1 / s)$ & $\mathbf{R}^{2}$ & Ko Fold Shift & $K_{D}(M)$ & Kon (1/Ms) & $k_{\text {off }}(1 / s)$ & $\mathbf{R}^{2}$ & Ko Fold Shift \\
\hline wt & $2.53 \mathrm{E}-10$ & $1.32 E+06$ & $2.78 \mathrm{E}-04$ & 0.99 & 1 & $<1 \mathrm{E}-12$ & $2.79 E+05$ & $<1 \mathrm{E}-07$ & 0.99 & 1 \\
\hline N74K & $2.98 \mathrm{E}-10$ & $1.01 \mathrm{E}+06$ & 3.02E-04 & 0.99 & 1.18 & $<1 \mathrm{E}-12$ & $3.33 E+05$ & $<1 \mathrm{E}-07$ & 0.99 & 1 \\
\hline R346I & $1.66 \mathrm{E}-10$ & $1.59 \mathrm{E}+06$ & 2.64E-04 & 0.99 & 0.66 & $7.89 \mathrm{E}-09$ & $2.12 \mathrm{E}+05$ & 1.68E-03 & 0.99 & 7,890 \\
\hline K444R & $2.31 \mathrm{E}-10$ & $1.66 \mathrm{E}+06$ & 3.82E-05 & 0.98 & 0.91 & $1.70 \mathrm{E}-08$ & $1.18 \mathrm{E}+05$ & $2.00 \mathrm{E}-03$ & 0.91 & 17,000 \\
\hline K444E & 1.77E-10 & $3.83 E+06$ & $2.76 \mathrm{E}-04$ & 0.99 & 0.7 & NB & NB & NB & NB & NB \\
\hline S686G & $1.80 \mathrm{E}-10$ & $1.63 E+06$ & 3.93E-04 & 0.99 & 0.71 & $<1 \mathrm{E}-12$ & $4.22 \mathrm{E}+05$ & $<1.0 \mathrm{E}-07$ & 0.99 & 1 \\
\hline
\end{tabular}

Extended Data Fig. 8 | Antibody resistant mutants selected with VSV-SARS-CoV2 or authentic SARS-CoV-2 virus. a. The method for assessing monoclonal antibody resistant spike protein variants is shown. SARS-CoV-2 was passaged serially in the presence of monoclonal antibodies at the increasing concentrations indicated in the figure or without antibody (no monoclonal antibody). Following passage at IC ${ }_{90}$ concentrations, samples were treated with $10 \times I_{90}$ concentrations of monoclonal antibodies and any resultant resistant virus collected, and the genome was sequenced. Red viruses in the schematic represent selection of escape variants. b. The escape phenotype of 6 independent plaques selected with AZD1061 was validated by demonstration of escape by testing in a PRNT. Antibody neutralization as measured by PRNT against the 6 plaque-purified, AZD1061-resistant SARS-CoV-2 viruses (blue) was compared to the parent virus WA-1 (orange) during treatment with AZD1061 or AZD7442. All plaque-purified viruses resulted from the same monoclonal antibody passage as detailed in (a). Data shown are from a single technical replicate for each of the six selected escape mutants. c,d. The escape phenotype of independent plaques selected with AZD1061 also was validated by demonstration of loss of binding to proteins incorporating variant residues in the selected plaques using biolayer interferometry (BLI). Data shown are from a single experiment. c. Binding traces of AZD8895 and AZD1061 to various spike trimers with kinetics curve fits. An inability to fit AZD1061 binding to the K444E S variant is due to a lack of detectable binding even at $5 \mu \mathrm{M}$ for AZD1061. d. Summary of AZD8895 and AZD1061 kinetic binding values to the S trimer variants from binding traces with $\mathrm{R}^{2}$ indicating goodness of the fit. Relative fold-change in $\mathrm{K}_{\mathrm{D}}$ is shown in comparison to wild-type. No detectable binding is indicated as NB. 


\section{Reporting Summary}

Nature Portfolio wishes to improve the reproducibility of the work that we publish. This form provides structure for consistency and transparency in reporting. For further information on Nature Portfolio policies, see our Editorial Policies and the Editorial Policy Checklist.

\section{Statistics}

For all statistical analyses, confirm that the following items are present in the figure legend, table legend, main text, or Methods section.

n/a Confirmed

$\searrow$ The exact sample size $(n)$ for each experimental group/condition, given as a discrete number and unit of measurement

$\searrow$ A statement on whether measurements were taken from distinct samples or whether the same sample was measured repeatedly

The statistical test(s) used AND whether they are one- or two-sided

$\triangle \square$ Only common tests should be described solely by name; describe more complex techniques in the Methods section.

\ $\square$ A description of all covariates tested

$\square$ \ A description of any assumptions or corrections, such as tests of normality and adjustment for multiple comparisons

$\square$ A full description of the statistical parameters including central tendency (e.g. means) or other basic estimates (e.g. regression coefficient)

AND variation (e.g. standard deviation) or associated estimates of uncertainty (e.g. confidence intervals)

For null hypothesis testing, the test statistic (e.g. $F, t, r$ ) with confidence intervals, effect sizes, degrees of freedom and $P$ value noted

Give $P$ values as exact values whenever suitable.

Х $\square$ For Bayesian analysis, information on the choice of priors and Markov chain Monte Carlo settings

Х $\square$ For hierarchical and complex designs, identification of the appropriate level for tests and full reporting of outcomes

Х $\square$ Estimates of effect sizes (e.g. Cohen's $d$, Pearson's $r$ ), indicating how they were calculated

Our web collection on statistics for biologists contains articles on many of the points above.

\section{Software and code}

Policy information about availability of computer code

Data collection We used the web-based data collection system at LS-CAT for remote data collection (https://ls-cat.org/dataCollection.html).

Data analysis The diffraction data were processed with XDS (doi:10.1107/S0907444909047337) and CCP4 suite (doi:10.1107/S0907444910045749 . The crystal structures were solved by molecular replacement using the structure of RDB in complex with Fab CC12.1 (PDB ID 6XC2) and Fab structure of MR78 (PDB ID 5JRP) with the program Phaser (doi:10.1107/S0021889807021206). The structures were refined and rebuilt manually with Phenix (doi:10.1107/S0907444909052925) or Coot ( doi:10.1107/S090744490401915), respectively. This study used commercially available GraphPad Prism software v8 for data representation and statistical analysis (GraphPad Prism; RRID: SCR_002798). UCSF chimera was used for molecular docking to the electron microscopy maps (UCSF Chimera; RRID: SCR_004097). PyMOL was used to visualize molecular structures and freely available from https://www.pymol.org/2//.The computational pipeline for the deep mutational scanning analysis of antibody escape mutations is available on GitHub: https://github.com/jbloomlab/SARS-CoV-2-RBD_MAP_AZ_Abs. The FASTQ files are available on the NCBI Sequence Read Archive under BioSample SAMN17532001 as part of BioProject PRJNA639956.. Permutation escape fractions are available on GitHub (https://github.com/jbloomlab/SARS-CoV-2-RBD_MAP_AZ_Abs/blob/main/results/ supp_data/AZ_cocktail_raw_data.csv) and in Supplementary Data Table 1. Flow cytometry data were analyzed using BD FACSDiva ${ }^{\mathrm{TM}}$ Software v6.1 (https://www.bdbiosciences.com/en-eu/products/software/instrument-software/bd-facsdiva-software). VSV-SARS-CoV-2 escape selection neutralization data were anallyzed using RTCA software version 2.1.0 (Agilent/Acea Biosciences; RRID: SCR_014821).

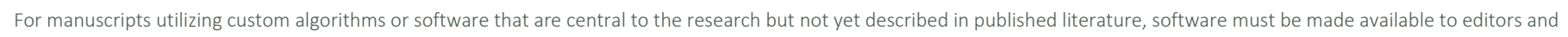

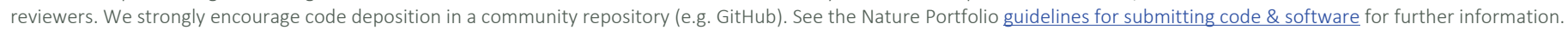


Policy information about availability of data

All manuscripts must include a data availability statement. This statement should provide the following information, where applicable:

- Accession codes, unique identifiers, or web links for publicly available datasets

- A description of any restrictions on data availability

- For clinical datasets or third party data, please ensure that the statement adheres to our policy

The crystal structures reported in this paper have been deposited to the Protein Data Bank (https://www.rcsb.org) under the accession numbers 7L7D (AZD8895 + RBD) and 7L7E AZD8895 and AZD1061 + RBD). The following were obtained from the PDB and used for visualization or molecular replacement: PDB IDs:7K4N, 6M0J, 6XM4, 78KW, 78KZ, 7CAK, 6ZOY, 6XC2, 5JRP. The FASTQ files for deep mutational scanning are available on the NCBI Sequence Read Archive under BioSample SAMN17532001 as part of BioProject PRJNA639956. Per-mutation escape fractions are available on GitHub (https://github.com/jbloomlab/SARS-CoV-2RBD_MAP_AZ_Abs/blob/main/results/supp_data/AZ_cocktail_raw_data.csv).

\section{Field-specific reporting}

Please select the one below that is the best fit for your research. If you are not sure, read the appropriate sections before making your selection. $\bigotimes$ Life sciences $\quad \square$ Behavioural \& social sciences $\quad \square$ Ecological, evolutionary \& environmental sciences For a reference copy of the document with all sections, see nature.com/documents/nr-reporting-summary-flat.pdf

\section{Life sciences study design}

All studies must disclose on these points even when the disclosure is negative.

Sample size Sample-size calculations were not performed to power each study. Key experiments that included in vitro measurements of antibody binding, and virus neutralizing activities were carried out with two or more independent study replicates, which were sufficient given the minimal differences observed in neutralization capacity of AZD1061 and AZD8895 against SARS-CoV-2 variants of concern/variants of interest.

Data exclusions No data were excluded from analysis.

Replication Studies that were repeated are noted in figure captions and include all studies that demonstrated the key results reported in the manuscript. No studies have been reported that failed upon repetition. Antibodies of known activity were included across all experiments to verify reproducibility (e.g. presence of binding, blocking, or neutralizing activities), and included comparisons of known SARS-CoV-2-specific antibodies to relevant characterized antibodies from the literature. These controls were included in each replicate experiment that measured binding and neutralizing activity of characterized anti-SARS-CoV-2 mAbs. Consistency of mAb activity across in vitro experiments within this study indicate a high level of reproducibility.

Randomization The principle of randomization is not relevant as this is an observational study.

Blinding The investigators were not blinded for most studies. We used conventional antigen binding and virus neutralization assays using actual binding to the SARS-CoV2 spike antigen and live SARS-CoV2 neutralization as the readouts.

\section{Reporting for specific materials, systems and methods}

We require information from authors about some types of materials, experimental systems and methods used in many studies. Here, indicate whether each material, system or method listed is relevant to your study. If you are not sure if a list item applies to your research, read the appropriate section before selecting a response.

Materials \& experimental systems

$\mathrm{n} / \mathrm{a}$ Involved in the study

Methods

$\square$ Antibodies

$\square$ 区ukaryotic cell lines

\ $\square$ Palaeontology and archaeology

$\mathrm{n} / \mathrm{a}$ Involved in the study

Х $\square$ chIP-seq

$\square$ X Flow cytometry

\ $\square$ Animals and other organisms

Х $\square$ MRI-based neuroimaging

\ $\square$ Human research participants

$\square$ Clinical data

$\square \bowtie$ Dual use research of concern

\section{Antibodies}

Antibodies used

Polyclonal goat anti-human IgG-HRP antibody (Southern Biotech Cat\# 2040-05) was used for antigen binding ELISA assays. For FRNT 

detection was performed using anti-human IgG ( $\gamma$-chain specific)-peroxidase antibody produced in goat (Sigma-Aldrich Cat\# A6029). A FITC-conjugated anti-MYC antibody (Immunology Consultants Lab, CYMC-45F. 1:100 dilution) was used to stain yeast to label RBD in flow cytometric assays, while a PE-conjugated goat anti-human-IgG (Jackson ImmunoResearch 109-115-098, 1:200 dilution) was used to label bound antibody. Previously discovered SARS-CoV2 spike antigen-specific monoclonal antibodies were described in PMID: 32668443

Validation

Previously discovered SARS-CoV2 spike antigen-specific monoclonal antibodies were validated via antigen binding, virus neutralization, and in a previous paper in vivo protection studies (PMID: 32668443). All other antibodies are commercially available. Antibodies used in a specific species or application have been appropriately validated by manufacturers and this information is provided on their website and information datasheets as follows:

Goat anti-human IgG-HRP (https://www.southernbiotech.com/?catno=2040-05\&type=Polyclonal\#\&panel1-1\&panel2-1); Anti-human IgG ( $\psi$-chain specific)-peroxidase antibody produced in goat (https://www.sigmaaldrich.com/content/dam/sigma-aldrich/ docs/Sigma/Datasheet/6/a6029dat.pdf);

FITC-conjugated anti-MYC antibody (https://www.icllab.com/anti-c-myc-antibody-chicken-fitc-13661.html);

PE-conjugated goat anti-human-IgG (https://www.jacksonimmuno.com/catalog/products/109-115-098)

\section{Eukaryotic cell lines}

Policy information about cell lines

Cell line source(s)

In this study we used the following cell lines: Vero E6 (American Type Culture Collection (ATCC), Cat\# CRL-1586), Vero (ATCC Cat\# CCL-81), HEK293 (ATCC Cat\# CRL-1573), and HEK293T (ATCC Cat\# CRL-3216), Expi293F (ThermoFisher Scientific, A1452), FreeStyle 293-F (ThermoFisher Scientific, R79007), and ExpiCHO (ThermoFisher Scientific, A29127). Vero-furin cells were obtained from T. Pierson (NIH) and have been previously described (PMID: 27420797).

Authentication

None of the cell lines used were authenticated.

Mycoplasma contamination

All cell lines were tested and confirmed negative for mycoplasma contamination.

Commonly misidentified lines

(See ICLAC register)

None

\section{Dual use research of concern}

Policy information about dual use research of concern

\section{Hazards}

Could the accidental, deliberate or reckless misuse of agents or technologies generated in the work, or the application of information presented in the manuscript, pose a threat to:

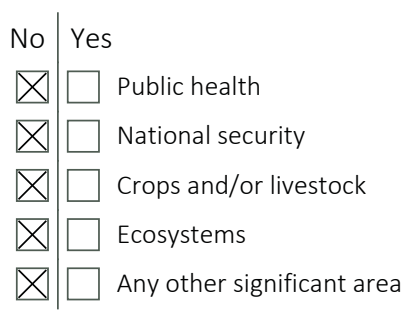

\section{Experiments of concern}

Does the work involve any of these experiments of concern:
No $\mid$ Yes
Х $\square$ Demonstrate how to render a vaccine ineffective
$\square$ \ Confer resistance to therapeutically useful antibiotics or antiviral agents
$\bigotimes \square$ Enhance the virulence of a pathogen or render a nonpathogen virulent
Х $\square$ Increase transmissibility of a pathogen
$\bigotimes \square$ Alter the host range of a pathogen
\ $\square$ Enable evasion of diagnostic/detection modalities
$\bigotimes \square$ Enable the weaponization of a biological agent or toxin
\ $\square$ Any other potentially harmful combination of experiments and agents 
Biosecurity oversight

All procedures were reviewed by standing university committees.

Benefits

Generation of escape mutant viruses was specifically requested by prior reviewers, is a conventional approach to defining the epitope and mechanism of action of neutralizing antibodies, and has important implications for the potential use of clinical-candidate monoclonal antibodies described in this manuscript.

Communication benefits Defining critical residues in this way is often requested by regulators to understand the potential for natural resistance, and as a means to monitor the emergence of resistance. Such studies are common in the field, and have been reviewed many times and not considered DURC. The antibodies in the current study are not licensed products nor do they have EUA, to date.

\section{Flow Cytometry}

\section{Plots}

Confirm that:

Х The axis labels state the marker and fluorochrome used (e.g. CD4-FITC).

\The axis scales are clearly visible. Include numbers along axes only for bottom left plot of group (a 'group' is an analysis of identical markers).

$\bigotimes$ All plots are contour plots with outliers or pseudocolor plots.

\ A numerical value for number of cells or percentage (with statistics) is provided.

\section{Methodology}

Sample preparation

Instrument

Software

Cell population abundance

Gating strategy

Tick this box to confirm that a figure exemplifying the gating strategy is provided in the Supplementary Information.

\begin{abstract}
Briefly, mutant yeast libraries induced to express RBD were washed and incubated with antibody at $400 \mathrm{ng} / \mathrm{mL}$ for $1 \mathrm{~h}$ at room temperature with gentle agitation. After the antibody incubations, the libraries were secondarily labeled with 1:100 FITC-conjugated anti-MYC antibody (Immunology Consultants Lab, CYMC-45F) to label for RBD expression and 1:200 PEconjugated goat anti-human-IgG (Jackson ImmunoResearch 109-115-098) to label for bound antibody. Flow cytometric sorting was used to enrich for cells expressing RBD variants with reduced antibody binding via a selection gate drawn to capture unmutated SARS-CoV-2 cells labeled at $1 \%$ the antibody concentration of the library samples.
\end{abstract}

BD LSRFortessa X-50

BD FACSDiva ${ }^{\mathrm{TM}}$ Software v6.1

Each mutant RBD library was labeled with $400 \mathrm{ng} / \mathrm{mL}$ (1x) antibody, and cells that were captured in the "antibody-escape bin" were sorted and their barcodes were sequenced. For a typical sample, approximately $15 \%$ of each sample fell into the "antibody-escaped" gate, compared with $<0.5 \%$ of the unmutated wild-type population stained with the same concentration of antibody. For each sample, approximately 10 million RBD+ cells were processed on the cytometer.

First, hierarchical gates were drawn to select single-cell events: forward scatter (FSC) versus side scatter (SSC), then SSC width versus height, and finally FSC width versus height. Next, FITC+ labeling of a C-terminal MYC epitope tag on the RBD was used to identify RBD+ cells. Selection gates for antibody-escaped sorts are nested within this RBD+ population. Unmutated SARS-CoV-2 RBD was labeled at $400 \mathrm{ng} / \mathrm{mL}(1 \mathrm{x})$ and $4 \mathrm{ng} / \mathrm{mL}(0.01 \mathrm{x})$ with each antibody. Antibody-escape selection gates were drawn to capture $0.5 \%$ or less of the $1 x$ and up $95 \%$ of the $0.01 x$ antibody-labeled unmutated RBD control cells. 